\title{
How Hard Is Bribery in Elections?
}

Piotr Faliszewski

Department of Computer Science

AGH University of Science and Technology

Kraków, Poland

Edith Hemaspaandra

Department of Computer Science

Rochester Institute of Technology

Rochester, NY 14623 USA

Lane A. Hemaspaandra

Department of Computer Science

University of Rochester

Rochester, NY 14627 USA
FALISZEW@AGH.EDU.PL

EH@CS.RIT.EDU

LANE@CS.ROCHESTER.EDU

\begin{abstract}
We study the complexity of influencing elections through bribery: How computationally complex is it for an external actor to determine whether by paying certain voters to change their preferences a specified candidate can be made the election's winner? We study this problem for election systems as varied as scoring protocols and Dodgson voting, and in a variety of settings regarding homogeneous-vs.-nonhomogeneous electorate bribability, bounded-size-vs.-arbitrary-sized candidate sets, weighted-vs.-unweighted voters, and succinct-vs.-nonsuccinct input specification. We obtain both polynomial-time bribery algorithms and proofs of the intractability of bribery, and indeed our results show that the complexity of bribery is extremely sensitive to the setting. For example, we find settings in which bribery is NP-complete but manipulation (by voters) is in P, and we find settings in which bribing weighted voters is NP-complete but bribing voters with individual bribe thresholds is in P. For the broad class of elections (including plurality, Borda, $k$ approval, and veto) known as scoring protocols, we prove a dichotomy result for bribery of weighted voters: We find a simple-to-evaluate condition that classifies every case as either NP-complete or in P.
\end{abstract}

\section{Introduction}

This paper studies the complexity of bribery in elections, that is, the complexity of computing whether it is possible, by modifying the preferences of a given number of voters, to make some preferred candidate a winner.

Election systems provide a framework for aggregating voters' preferences - ideally (though there is no truly ideal voting system, see Duggan \& Schwartz, 2000; Gibbard, 1973; Satterthwaite, 1975) in a way that is satisfying, attractive, and natural. Societies use elections to select their leaders, establish their laws, and decide their policies, but practical applications of elections are not restricted to people and politics. Many parallel algorithms start by electing leaders. Multiagent systems sometimes use voting for the purpose of planning (Ephrati \& Rosenschein, 1997). Web search engines can aggregate results using methods based on elections (Dwork, Kumar, Naor, \& Sivakumar, 2001). With such a wide 
range of applications, it is not surprising that elections vary tremendously. For example, one might think at first that typical elections have many voters and very few candidates. However, in fact, they may have a very wide range of voter-to-candidate proportions: In typical presidential elections there are relatively few candidates but there may be millions of voters. In the context of the web, one may consider web pages as voting on other pages by linking to them, or may consider humans to be voting on pages at a site by the time they spend on each. In such a setting we may have both a large number of voters and a large number of candidates. On the other hand, Dwork et al. (2001) suggest designing a meta search engine that treats other search engines as voters and web pages as candidates. This yields very few voters but many candidates. To summarize this paragraph, elections have a great variety of sizes in terms of numbers of candidates and numbers of voters. So, surely, one should not simply say "Elections tend to be small, and so we can always solve by brute-force the issues related to them."

With the principles of democracy in mind, we also tend to think that each vote is equally important. However, all the above scenarios make just as much sense in a setting in which each voter has a different voting power. For example, U.S. presidential elections are in some sense weighted (different states have different voting powers in the Electoral College); shareholders in a company have votes weighted by the number of shares they own; and search engines in the above example could be weighted by their quality. Weighted voting is a natural choice in many other settings as well.

The importance of election systems naturally inspired questions regarding their resistance to abuse, and several potential dangers were identified and studied. For example, an election's organizers can make attempts to control the outcome of the elections by procedural tricks such as adding or deleting candidates or encouraging/discouraging people from voting. Classical social choice theory is concerned with the possibility or impossibility of such procedural control. However, recently it was realized that even if control is possible, it may still be difficult to find what actions are needed to effect control, e.g., because the computational problem is NP-complete. The complexity of controlling who wins the election was studied first by Bartholdi, Tovey, and Trick (1992) and later on by many other authors (Faliszewski, Hemaspaandra, Hemaspaandra, \& Rothe, 2007; Hemaspaandra, Hemaspaandra, \& Rothe, 2007; Erdélyi, Nowak, \& Rothe, 2008a, 2008b; Faliszewski, Hemaspaandra, Hemaspaandra, \& Rothe, 2008; Meir, Procaccia, Rosenschein, \& Zohar, 2008). Elections are endangered not only by the organizers but also by the voters (manipulation), who might be tempted to vote strategically (that is, not according to their true preferences) to obtain their preferred outcome. This is not desirable as it can skew the result of the elections in a way that is arguably not in the best interest of the society. The GibbardSatterthwaite/Duggan-Schwartz Theorems (Gibbard, 1973; Satterthwaite, 1975; Duggan \& Schwartz, 2000) show that essentially all election systems can be manipulated, and so it is important to discover for which systems manipulation is computationally difficult to execute. This line of research was started by Bartholdi, Tovey, and Trick (1989a) and was continued by many researchers (as a few varied examples, we mention the work of Elkind \& Lipmaa, 2005b, 2005a; Conitzer, Sandholm, \& Lang, 2007; Hemaspaandra \& Hemaspaandra, 2007; Procaccia \& Rosenschein, 2007; Brelsford, Faliszewski, Hemaspaandra, Schnoor, \& Schnoor, 2008; Faliszewski, Hemaspaandra, \& Schnoor, 2008; and Zuckerman, Procaccia, \& Rosenschein, 2008; readers interested in manipulation will be able to reach a broader collec- 
tion of papers through the standard process of recursive bibliography search). Faliszewski, Hemaspaandra, Hemaspaandra, and Rothe (2009b) provide a relatively nontechnical survey of some complexity issues related to altering the outcomes of elections.

Surprisingly, nobody seems to have addressed the issue of (the complexity of) bribery, i.e., attacks where the person interested in the success of a particular candidate picks a group of voters and convinces them to vote as he or she says. Bribery seems strongly motivated by both real-life and computational agent-based settings, and shares some of the flavor of both manipulation (changing voters' (reported) preferences) and control (deciding which voters to influence). This paper (in this version and its conference precursor) initiates the study of the complexity of bribery in elections.

There are many different settings in which bribery can be studied. In the simplest one we are interested only in the least number of voters we need to bribe to make our favored candidate win. A natural extension is to consider prices for each voter. In this setting, each voter is willing to change his or her true preferences to anything we say, but only if we can meet his or her price. In an even more complicated setting it is conceivable that voters would have different prices depending on how we want to affect their vote (however, it is not clear how to succinctly encode a voter's price scheme). We mainly focus on the previous two scenarios but we do point the reader to our results on approval voting and to the work of Faliszewski (2008) for a discussion of bribery when prices are represented more flexibly.

We classify election systems with respect to bribery by seeking in each case to either (a) prove the complexity is low by giving a polynomial-time algorithm or (b) argue intractability via proving the NP-completeness of discovering whether bribery can affect a given case. We obtain a broad range of results showing that the complexity of bribery depends closely on the setting. For example, for weighted plurality elections bribery is in $\mathrm{P}$ (Theorem 3.3) but jumps to being NP-complete if voters have price tags (Theorem 3.2). As another example, for approval voting the manipulation problem is easily seen to be in $\mathrm{P}$, but in contrast we prove that the bribery problem is NP-complete (Theorem 4.2). Yet we also prove that when the bribery cost function is made more local, the complexity of approval voting falls back to $\mathrm{P}$ (Theorem 4.4). But if more constraints are added, the problem goes up to NP-complete again (Theorem 4.5). For scoring protocols we obtain complete characterizations of the complexity of bribery for all possible voter types, i.e., with and without weights and with and without price tags. In particular, via dichotomy theorems (Theorems 4.8 and 4.9) and algorithmic constructions (Theorems 4.13 and 4.15) we provide for each voter type a simple condition that partitions all scoring protocols into ones with NP-complete bribery problems and ones with $\mathrm{P}$ bribery problems. We point the reader to Tables 1 and 2 (they appear near the end of this paper) for a summary of some of the complexity results regarding bribery in elections.

The paper is organized as follows. In Section 2 we describe the election systems and bribery problems we are interested in and we cover some complexity background and preliminaries. In Section 3, we provide a detailed study of plurality elections. After that we study connections between manipulation and bribery, and obtain dichotomy results for bribery under scoring protocols in Section 4. In Section 5, we study the case of succinctly represented elections with a fixed number of candidates. 


\section{Preliminaries}

This section introduces the notions and notations that we use in the paper.

\subsection{Election Systems}

We describe elections by providing a set $C=\left\{c_{1}, \ldots, c_{m}\right\}$ of candidates, a set $V$ of $n$ voters specified by their preferences, and a rule for selecting winners. A voter $v$ 's preferences are represented as a list $c_{i_{1}}>c_{i_{2}}>\ldots>c_{i_{m}},\left\{i_{1}, i_{2}, \ldots, i_{m}\right\}=\{1,2, \ldots, m\}$, where $c_{i_{1}}$ is the most preferred candidate and $c_{i_{m}}$ is the most despised one. We assume that preferences are transitive, complete (for every two candidates each voter knows which one he or she prefers), and strict (no ties). Sometimes authors also allow ties in the preference lists, but ties do not have a clear interpretation for some election rules and so for simplicity and uniformity we do not consider them.

Given a list of votes (i.e., of voters' preference lists), an election rule determines which candidates are winners of the elections. We now briefly describe the election systems that we analyze in this paper, all of which are standard in the literature on social choice theory. ${ }^{1}$ Winners of plurality elections are the candidate(s) who are the top choice of the largest number of voters (of course, these will be different voters for different winners). In approval voting each voter selects candidates he approves of; the candidate(s) with the most approvals win. Unlike all the other systems discussed in this paper, under approval voting the input is not a preference order but rather is a bit-vector of approvals/disapprovals. A scoring protocol for $m$ candidates is described by a vector $\alpha=\left(\alpha_{1}, \ldots, \alpha_{m}\right)$ of nonnegative integers such that $\alpha_{1} \geq \alpha_{2} \ldots \geq \alpha_{m}$. (We do not require $\alpha_{1}>\alpha_{m}$, as we wish to classify the broadest class of cases possible, including the usually easy boundary case when all $\alpha_{i}$ 's are equal.) Each time a candidate appears in the $i$ 'th position of a voter's preference list, that candidate gets $\alpha_{i}$ points; the candidate(s) who receive the most points win. Wellknown examples of scoring protocols include the Borda count, plurality, $k$-approval, and veto voting systems, where for $m$-candidate elections Borda uses $\alpha=(m-1, m-2, \ldots$, $0)$, plurality uses $\alpha=(1,0, \ldots, 0,0), k$-approval uses $\alpha=(\overbrace{1, \ldots, 1}^{k}, \overbrace{0, \ldots, 0}^{m-k})$, and veto uses $\alpha=(1,1, \ldots, 1,0)$. Note that by selecting a scoring protocol we automatically select the number of candidates we have within elections. Though some scoring protocols can easily and naturally be generalized to arbitrary candidate sets, formally each individual scoring protocol deals with only a fixed number of candidates. Thus all our results regarding scoring protocols automatically talk about a fixed number of candidates.

A Condorcet winner is a candidate who (strictly) beats all other candidates in pairwise contests, that is, a Condorcet winner beats everyone else in pairwise plurality elections. Clearly, there can be at most one Condorcet winner, but sometimes there are none (as is the case in the Condorcet Paradox, Condorcet, 1785). There are many voting systems that

1. In the social choice literature, often voting systems are assumed to have at least one winner, or exactly one winner, but at least in terms of the basic definition of a voting system, we do not require such a restriction, since one can imagine wanting to study elections in which-perhaps due to tie effects or symmetry effects or perhaps even due to having zero candidates - there is not always exactly one winner. Indeed, in practice, in such elections as those on Hall of Fame induction worthiness or on who should be hired at a given academic department, it is quite possible that a real-world election system might give the answer "No one this year." 
choose the Condorcet winner if one exists and use some compatible rule otherwise. One such system - developed in the 1800s - is that of Charles Lutwidge Dodgson (a.k.a. Lewis Carroll). In Dodgson's system a winner is the person(s) who can become a Condorcet winner by the smallest number of switches in voters' preference lists. (A switch changes the order of two adjacent candidates on a list. ${ }^{2}$ ) Thus, if a Condorcet winner exists, he or she is also the unique winner of Dodgson's election. See the work of Dodgson (1876) - and also the work of Bartholdi, Tovey, and Trick (1989b) — for details regarding Dodgson's voting rule, under which it is now known that winner testing is complete for parallel access to NP (Hemaspaandra, Hemaspaandra, \& Rothe, 1997). A different election rule was introduced more recently by Young (1977). In Young elections a winner is a person who can become a Condorcet winner by removing the smallest number of voters. By way of contrast, note that plurality rule has the property that it elects those candidates who, after removing the least number of votes, are preferred by everyone. The work of Rothe, Spakowski, and Vogel (2003), see also the expository presentation of Rothe (2005), proves that the winner problem in Young elections is extremely difficult - complete for parallel access to NP.

Another election rule is that of Kemeny (1959), see also the work of Kemeny and Snell (1960). A Kemeny consensus is a preference order that maximizes the number of agreements with voters' preference lists, where for each voter and for each two candidates $a$ and $b$ we say that a preference order agrees with a voter's preference list if both place $a$ below $b$ or both place $b$ below $a$. Naturally, many different Kemeny consensuses may be possible. A candidate is a winner in a Kemeny election if he or she is the most preferred candidate in some Kemeny consensus of that election. (The original work of Kemeny allowed voters to have nonstrict preference orders, but like, e.g., Saari \& Merlin, 2000, we use Kemeny elections to refer to just the case where input orderings are strict.) Note that the winner testing problem for Kemeny elections is known to be complete for parallel access to NP, and this is known to hold both in the case when input preference orders must be strict, and in the case when nonstrict input preference orders are allowed (Hemaspaandra, Spakowski, \& Vogel, 2005, and see in particular the comments in the footnote on page 383 of that paper). The Kemeny rule might at first sound as if it were the same as the Dodgson rule, but in fact they are very different. Dodgson's elections are based on making the minimum number of local changes, but Kemeny's elections hinge on the overall closeness of the voters' preference orders to certain "consensus" orderings - which themselves possibly may not be the preferences of any of the voters.

\subsection{Bribery Problems}

Informally, the bribery problem is the following: Given the description of an election (i.e., the set of candidates, the preferences of the voters, etc.), a number $k$, and some distinguished candidate $p$, can we make $p$ a winner by changing the preference lists of at most $k$ voters. More formally, for an election rule (i.e., election system) $\mathcal{E}$ we define the $\mathcal{E}$-bribery problem to be the following. We assume a standard encoding of mathematical objects such as finite

2. We mention, since this can be a source of confusion, that in his seminal paper Dodgson did not explicitly state that switches were limited to adjacent candidates. However, the mathematics of his examples is consistent with only that reading, and so it is clear that that is his intended meaning. 
sets and lists (e.g., see Garey \& Johnson, 1979). Also, all our numbers will be nonnegative integers and, unless otherwise specified, will be represented in binary.

Name: $\mathcal{E}$-bribery.

Given: A set $C$ of candidates, a collection $V$ of voters specified via their preference lists. A distinguished candidate $p \in C$ and a nonnegative integer $k$.

Question: Is it possible to make $p$ a winner of the $\mathcal{E}$ election by changing the preference lists of at most $k$ voters?

We will speak both of the unweighted case (all voters are equal; in this paper that always holds unless "weighted" is in the problem name) and the weighted case (voters are weighted). Essentially all our results apply both to the case in which we want to make the preferred candidate a winner and to the case in which we want to make the preferred candidate the unique winner, and so we have not explicitly put a nonunique/unique setting into the problem names. For clarity and specificity, we focus on the nonunique case in our discussions and proofs, and all our problem statements and theorems by default refer to the nonunique case. However, in most settings the differences between the proofs for the unique case and the nonunique case are very minor and amount to a couple of small tweaks, e.g., changing weak inequalities to strong ones, adding a special voter who already prefers $p$, etc., and we often at the end of a proof briefly note that the theorem also holds for the unique case.

In the $\mathcal{E}$-\$bribery family of problems we assume that each voter has a price for changing his or her preference list. In such a case we ask not whether we can bribe at most $k$ people, but whether we can make $p$ a winner by spending at most $k$ dollars. For example, the plurality-weighted-\$bribery problem can be described as follows.

Name: plurality-weighted-\$bribery.

Given: A set $C$ of candidates. A collection $V$ of voters specified via their preference lists $\left(\right.$ prefs $_{1}, \ldots$, prefs $\left._{m}\right)$, their (nonnegative, integer) weights $\left(w_{1}, \ldots, w_{m}\right)$, and their (nonnegative, integer) prices $\left(p_{1}, \ldots, p_{m}\right)$. A distinguished candidate $p \in C$ and a nonnegative integer $k$ (which we will sometimes refer to as the budget).

Question: Is there a set $B \subseteq\{1, \ldots, m\}$ such that $\sum_{i \in B} p_{i} \leq k$ and there is a way to bribe the voters from $B$ in such a way that $p$ becomes a winner?

Regarding the fact that in these models voters are assumed to vote as the bribes dictate, we stress that by using the term bribery we do not intend to necessarily imply any moral failure on the part of bribe recipients or bribe givers: Bribes are simply payments. Although in human, political elections, such payments are typically considered morally wrong (perhaps because each voter is supposed to be thinking of the overall social welfare), in electronic/web/multiagent systems settings, such morality issues often do not apply. A voter may simply be an electronic entity trying to maximize its utility, and the bribe price of that entity may crisply reflect that fact.

Throughout this paper we use the term bribery both in its regular sense and in the nonstandard sense of "a collection of bribes." We will when using the latter sense often speak of "a bribery," by which we thus mean a collection of bribes. So, for example, "if 
Alice can end up a winner by a bribery that does not ask anyone to vote for Alice," we mean "if there is a pattern/scheme of bribes (e.g., give two dollars to Bob to make the change FOO in his vote and give five dollars to Carol to make change BAR in her vote) none of which asks anyone to vote for Alice yet that make Alice the overall winner."

As we will be dealing with a variety of settings, we need some common format to speak of the instances of bribery problems. We adopt the following convention (and we view the already specified problems to be implicitly recast into this form): An instance of a bribery problem is a 4 -tuple $E=(C, V, p, k)$, where

1. $C$ is a list of candidates,

2. $V$ is a list of voters (see below),

3. $p \in C$ is the candidate that we want to make a winner (for problems about making a candidate a unique winner, the "a winner" here is replaced with "a unique winner"), and

4. $k$ is the bribe limit (either the amount of money we can spend on bribing or the maximum number of voters we can bribe, depending on the flavor of the bribery problem).

The list of voters contains tuples describing the votes that are cast. Each voter is a 3-tuple (prefs, $\pi, \omega)$, where

1. prefs is the preference list of the voter (or, in the case of approval voting, his or her preference vector),

2. $\pi$ is the price for changing this voter's preference list, and

3. $\omega$ is the weight of the voter.

Each tuple in $V$ describes precisely one voter. We drop the price and/or the weight field if in the given election the voters have no prices/weights. (However, we do assume that dropped prices and weights have unit values, so that we can refer to them. Some of our proofs handle two cases, one with priced voters and one with weighted voters, at the same time and need to be able to uniformly refer to both weights and prices.) If $v \in V$ is a voter then we refer to his or her price and weight as $\pi(v)$ and $\omega(v)$. In the same manner, if $U \subseteq V$ then

$$
\begin{aligned}
& \pi(U)=\sum_{v \in U} \pi(v) \text { and } \\
& \omega(U)=\sum_{v \in U} \omega(v) .
\end{aligned}
$$

We will often refer to $\omega(U)$ either as "the vote weight of $U$ " or as "the total weight of $U$."

Note that throughout this paper $V$, though input as a list, typically functions as a multiset, and so summations such as those above do have an additive term for each appropriate occurrence in the multiset - the multiplicities carry into such sums, and also into set-like 
operations, e.g., $\{v \in V \mid \ldots\}$ will itself be a multiset, with multiplicities appropriately preserved. And we when dealing with $V$ use set/subset to mean multiset/submultiset.

In Section 5 we deal with succinct representations. When we are dealing with succinct representations, $V$ will consist of 4 -tuples (prefs, $\pi, \omega, m$ ), where $m$ is the multiplicity of the vote, that is, a number of voters of identical preferences, price, and weight that this entry in $V$ is standing for. $m(v)$ will denote the $m$ value of a $v \in V$. Note that here single entry in $V$ often represents multiple voters.

This notation will help us speak of bribery problems in a uniform fashion. Note that in addition to specifying $E=(C, V, p, k)$ we always need to explicitly state what election rule we are using.

Positive results regarding more demanding bribery problems imply positive results about weaker ones. For example, if weighted bribery is in $\mathrm{P}$ for some election system $\mathcal{E}$ then clearly we have that unweighted bribery is also easy for $\mathcal{E}$. Conversely, hardness results regarding simpler models imply hardness results about the more involved ones. We often mention such "implied" results separately if they are interesting (e.g., if an algorithm for a simpler case provides insights for understanding the more complicated case), but we omit them if they are not enlightening.

\subsection{Reductions and NP-completeness}

Before we proceed with the study of bribery, let us briefly review some notions of computational complexity and some standard NP-complete problems that we will use in our proofs.

As usual, $\|S\|$ denotes the cardinality of the set $S$. We fix our alphabet to be $\Sigma=\{0,1\}$ and we assume standard encodings of mathematical entities involved in our problems. In particular, all integers are represented in binary unless specified otherwise. (For example, see Garey \& Johnson, 1979, for a discussion of these issues.) By NP-completeness we as is standard mean completeness with respect to many-one (polynomial-time) reductions.

Definition 2.1 $A \leq_{m}^{p} B$ (A many-one reduces to $B$ ) if there is a polynomial-time computable function $f$ such that

$$
\left(\forall x \in \Sigma^{*}\right)[x \in A \Longleftrightarrow f(x) \in B] .
$$

In one of our results relating manipulation and bribery we also need disjunctive truth-table reductions.

Definition 2.2 We say that $A \leq_{d t t}^{p} B$ ( $A$ disjunctively truth-table reduces to $B$ ) if there is a polynomial-time procedure that on input $x$ outputs a list of strings $y_{1}, \ldots, y_{m}$ such that $x \in A$ if and only if at least one of $y_{i}, 1 \leq i \leq m$, is in $B$.

Both of the above definitions are standard and commonly used within the field of complexity theory. Detailed treatment of various reduction types including these can be found, e.g., in the work of Ladner, Lynch, and Selman (1975).

A standard way of showing that a problem is NP-complete is by proving it is in NP and reducing some known NP-complete problem to it. The former is easy for most of the bribery problems that we deal with: If we can compute the winners of the elections in polynomial time, then we can just nondeterministically guess a bribe and test whether it yields the 
desired outcome. For the latter issue we use reductions from either the partition problem or the exact cover by 3-sets problem (e.g., see Garey \& Johnson, 1979; Papadimitriou, 1994, for general background on these problems and on proving NP-completeness).

The problem Partition asks whether it is possible to split a sequence of integers into two subsequences that have equal sums.

Name: Partition.

Given: A sequence $s_{1}, \ldots, s_{n}$ of nonnegative integers satisfying $\sum_{i=1}^{n} s_{i} \equiv 0(\bmod 2){ }^{3}$

Question: Is there a set $A \subseteq\{1, \ldots, n\}$ such that $\sum_{i \in A} s_{i}=\sum_{i \in\{1, \ldots, n\}-A} s_{i}$ ?

To prove our main dichotomy result in Section 4 we need a more restrictive version of the partition problem. Let $s_{1}, \ldots, s_{n}$ be a sequence of nonnegative integers such that $\sum_{i=1}^{n} s_{i} \equiv 0(\bmod 2)$. In Partition' we assume that for each $i, 1 \leq i \leq n$, it holds that

$$
s_{i} \geq \frac{1}{2+n} \sum_{i=1}^{n} s_{i}
$$

(reminder: footnote 3 of course applies regarding the handling of both $\sum_{i=1}^{n} s_{i} \equiv 0(\bmod 2)$ and $\left.(\forall i \in\{1, \ldots, n\})\left[s_{i} \geq \frac{1}{2+n} \sum_{i=1}^{n} s_{i}\right]\right)$, and we ask whether there exists an $A \subseteq\{1, \ldots, n\}$ such that $\sum_{i \in A} s_{i}=\frac{1}{2} \sum_{i=1}^{n} s_{i}$. For the sake of completeness we include a proof that Partition' remains NP-complete.

Lemma 2.3 Partition' is NP-complete.

Proof. Clearly, Partition' is in NP. We will show, by a reduction from the standard partition problem, that Partition' is also NP-hard.

Let $q=s_{1}, \ldots, s_{n}$ be a sequence of nonnegative integers and let $2 S=\sum_{i=1}^{n} s_{i}$. First, we construct a sequence $q^{\prime}=s_{1}^{\prime}, o_{1}^{\prime}, \ldots, s_{n}^{\prime}, o_{n}^{\prime}$ of $2 n$ nonnegative integers that has the following two properties. (1) $q^{\prime}$ can be partitioned if and only if $q$ can be. (2) Each partition of $q^{\prime}$ splits $q^{\prime}$ into two sequences of the same cardinality. We define $s_{i}^{\prime}$ and $o_{i}^{\prime}$, for $1 \leq i \leq n$, as follows.

$$
\begin{aligned}
s_{i}^{\prime} & =3^{i-1}+3^{n} s_{i} . \\
o_{i}^{\prime} & =3^{i-1} .
\end{aligned}
$$

Any partition of $s_{1}^{\prime}, o_{1}^{\prime}, \ldots, s_{n}^{\prime}, o_{n}^{\prime}$ splits $q^{\prime}$ into two subsequences that each sum up to $S^{\prime}$, where $S^{\prime}$ is defined as

$$
S^{\prime}=\frac{1}{2} \sum_{i=1}^{n}\left(s_{i}^{\prime}+o_{i}^{\prime}\right)=3^{n} S+\sum_{i=1}^{n} 3^{i-1}=3^{n} S+\frac{3^{n}-1}{2} .
$$

3. If for a given input it holds that $\sum_{i=1}^{n} s_{i} \not \equiv 0(\bmod 2)$, we consider the input to be "syntactically" illegal and thus consider that input not to be a member of Partition. For the rest of this paper we assume, when reducing from Partition to some other problem $(Q)$, that if some "syntactic" constraint is violated by the input, then our reduction will not do whatever the reduction we give states, but rather will instantly map to a fixed element of $\bar{Q}$. We (often tacitly) make the same assumption - that "syntactically" (by which we mean both true conditions of syntax and other polynomial-time constraints on a problem via the "Given" assumes apply to its inputs) illegal inputs are not handled via the reduction's operation on the input's components, but rather are mapped to a fixed element of the complement of the set being reduced to. 
Clearly, any partition of $s_{1}^{\prime}, o_{1}^{\prime}, \ldots, s_{n}^{\prime}, o_{n}^{\prime}$ splits $q^{\prime}$ into two halves such that if $s_{i}^{\prime}$ belongs to one then $o_{i}^{\prime}$ belongs to the other. It is also immediate that $q$ can be partitioned if and only if $q^{\prime}$ can.

To satisfy condition (1) we add a constant to each $s_{i}^{\prime}$ and $o_{i}^{\prime}$. Define $\widehat{q}$ to be a sequence of numbers $\widehat{s}_{1}, \widehat{o}_{1}, \ldots, \widehat{s}_{n}, \widehat{o}_{n}$ such that for each $i, 1 \leq i \leq n$,

$$
\begin{aligned}
\widehat{s}_{i} & =s_{i}^{\prime}+S^{\prime} \text { and } \\
\widehat{o}_{i} & =o_{i}^{\prime}+S^{\prime} .
\end{aligned}
$$

Clearly, any partition of $q^{\prime}$ still is a partition of $\widehat{q}$, since any partition of $q^{\prime}$ splits $q^{\prime}$ into two subsequences of the same cardinality. The converse holds because any partition of $\widehat{q}$ has to split it into subsequences that each sum up to $\widehat{S}=S^{\prime}+n S^{\prime}$ and this is only possible if each subsequence contains exactly $n$ elements. (A sum of more than $n$ elements would be greater than $(n+1) S^{\prime}$ and that would be more than the other subsequence could sum up to.) It remains to show that (1) holds for $\widehat{q}$. This is the case because each $\widehat{s}_{i}$ and $\widehat{o}_{i}$ is greater than $S^{\prime}$ and $S^{\prime}=\frac{2}{2+2 n} \widehat{S}$. (Note that sequence $\widehat{q}$ has $2 n$ elements.) Since $\widehat{q}$ can be computed in polynomial time, the proof is completed.

The exact cover by 3 -sets problem (X3C) asks about a way to pick, from a given list, three-element subsets of some set $B$ so as to cover the whole set without ever introducing the same element more than once.

Name: X3C.

Given: $\mathrm{A}$ set $B=\left\{b_{1}, \ldots, b_{3 t}\right\}$ and a family of three-element subsets of $B, S=$ $\left\{S_{1}, \ldots, S_{m}\right\}$.

Question: Is there a set $A \subseteq\{1, \ldots, m\}$ such that $\|A\|=t$ and $\bigcup_{i \in A} S_{i}=B$ ?

These two problems-Partition and $\mathrm{X} 3 \mathrm{C}$ - have been useful tools for proving NPcompleteness of control and manipulation problems, and in this paper we will see that they are very powerful when used for bribery problems. Specifically, Partition will be very useful when we are dealing with weighted elections and $\mathrm{X} 3 \mathrm{C}$ will be particularly useful in the unweighted cases.

\section{Plurality}

In this section we determine the complexity of bribery for plurality-rule elections. Plurality rule is perhaps the most popular election system in practical use; from the point of view of democracy it is very natural and appealing to make a decision that many people prefer. However, there are also downsides to plurality rule. Plurality rule may slight the voices of minorities and does not take into account full information about voters' preferences. In particular, if there is some candidate that all voters rank as second best and no other candidate is the top choice of many rankings, it might seem natural to elect this "second best" person. However, plurality is blind to this. In fact, we will typically view a vote in plurality-rule elections as a vote for a particular candidate, namely, the most preferred candidate according to the preference order that is the actual vote; for the purposes of this paper that is the only thing that matters about the voter, though we mention that in 
other contexts, such as "control" problems allowing deletion of candidates (Bartholdi et al., 1992; Hemaspaandra et al., 2007; Hemaspaandra, Hemaspaandra, \& Rothe, 2009), the full ordering might be important. The simplicity and widespread use of plurality-rule elections make the results of this section of particular relevance.

We in the previous section somewhat carelessly mentioned plurality as a scoring rule with the vector $\alpha=(1,0, \ldots, 0)$. Of course, formally speaking, what really holds is that for each number of candidates $k$ there is a notion of a plurality-of- $k$-candidates scoring-rule election, which has the vector that is a 1 followed by $k-10$ 's. But one can also consider the system that takes as input any number of candidates and then applies the plurality scoring rule (for that input's number of candidates). This is what most people think of when speaking of "plurality elections," and throughout this section, our results about plurality are of course about not just fixed numbers of candidates, but are about this case.

The simplest bribery scenario is when the voters are unweighted and each voter is as expensive to bribe as each other voter. Not surprisingly, bribery is easy in such a setting.

Theorem 3.1 plurality-bribery is in $\mathrm{P}$.

Proof. The proof of this theorem is simple, but we describe it in detail as a simple introduction to our proofs regarding bribery. We will give a polynomial-time algorithm that given an instance of bribery $E=(C, V, p, k)$ decides whether it is possible to make $p$ a winner by bribing at most $k$ voters.

Our algorithm works in the following way. Initially we have bribed zero voters. We check whether $p$ currently is a winner. If so, we accept. Otherwise, until doing so will exceed the bribe limit, we pick any current winner, bribe one of his or her voters (recall, as mentioned earlier in this section, that by "his or her [i.e., the selected winner's] voters" we mean those voters having that particular selected winner as their most preferred candidate) to vote for $p$, and jump back to testing whether $p$ is a winner. If we reach the bribe limit (i.e., in the above we have the "until doing so will exceed the bribe limit" break us out of the loop) without making $p$ a winner then we reject.

If this algorithm accepts then obviously bribery is possible. We now show that if it is possible to ensure that $p$ is a winner via at most $k$ bribes then our algorithm accepts. Our proof follows by induction on $k$. For the base case it is enough to note that the algorithm works correctly for $k=0$. For the induction step, let us assume that on each input $E^{\prime}=\left(C^{\prime}, V^{\prime}, p, k^{\prime}\right)$ such that $k^{\prime}<k$, where $k$ is some positive integer, the algorithm accepts exactly if it is possible to ensure that $p$ is a winner via at most $k^{\prime}$ bribes. Now, let $E=(C, V, p, k)$ be an arbitrary input such that $p$ can become a winner via at most $k$ bribes. We will show that our algorithm accepts this input. We consider two cases. If there is a bribery of up to $k$ voters that ensures $p$ 's victory but that never involves any of the voters of any of the current winners of election $(C, V)$ then it is clear that our algorithm accepts. (Let $V_{p} \subseteq V$ be the set of all voters who do not vote for $p$. In this case any bribery of $\min \left(k,\left\|V_{p}\right\|\right)$ voters ensures that $p$ becomes a winner.) Thus, let us assume that the only briberies that make $p$ a winner involve bribing at least one voter of one of the current winners. Let $c_{1}$ be one of the winners of $(C, V)$ (note that $\left.c_{1} \neq p\right)$ and let $E^{\prime \prime}=\left(C, V^{\prime \prime}, p, k-1\right)$ be an instance of plurality-bribery obtained from $E$ by bribing one of $c_{1}$ 's voters. Clearly, after executing a single iteration of the loop, our algorithm transforms its input into one that 
is isomorphic to $E^{\prime \prime}$. By our assumptions and by the inductive hypothesis the algorithm accepts its transformed input.

The algorithm works in polynomial time as at most $\|V\|$ bribes suffice to make $p$ a winner and each of the iterations can be executed in polynomial time. The theorem is proven. We mention that the same approach clearly also works for the unique case.

The ease of obtaining the above algorithm might fool us into thinking that bribery within the plurality system is always easy. However, that is not the case.

Theorem 3.2 plurality-weighted-\$bribery is NP-complete, even for just two candidates.

Proof. Recall that the nonunique version of the problem is our default case, and so we are addressing that here.

plurality-weighted-\$bribery is in NP: We can guess the voters to bribe and test whether such a bribe both makes our designated candidate a winner and does not exceed the budget. It remains to show that the problem is NP-hard.

To show NP-hardness, we will construct a reduction from Partition. Let $s_{1}, \ldots, s_{n}$ be a sequence of nonnegative integers and let $\sum_{i=1}^{n} s_{i}=2 S$. Our goal is to design an election $E=(C, V, p, k)$ in which $p$ can become a winner by bribery of cost at most $k$ if and only if there is a set $A \subseteq\{1, \ldots, n\}$ such that $\sum_{i \in A} s_{i}=S$. We define the election to have two candidates, $p$ and $c$, and exactly $n$ voters, $v_{1}, \ldots, v_{n}$, with each $v_{i}$ having both weight and price equal to $s_{i}$. All voters prefer $c$ to $p$. The budget $k$ is set to $S$. We claim that $p$ can become a winner if and only $s_{1}, \ldots, s_{n}$ can be partitioned into two equal-sum groups.

Let us assume that there is a set $A \subseteq\{1, \ldots, n\}$ such that $\sum_{i \in A} s_{i}=S$. This means that for each $i \in A$ we can bribe $v_{i}$ to vote for $p$ and get for $p$ a total vote weight (in the natural sense, as was defined in Section 2) of $S$. This makes $p$ a winner. On the other hand, assume that $p$ can be made a winner by bribes of total cost at most $k=S$. The weight of each voter is equal to his or her price and so $p$ can obtain at most vote weight $k=S$. In fact, $p$ must obtain exactly vote weight $S$, since from our setup it is clear that if $p$ gains strictly less than vote weight $S$ then $c$ will be the unique winner. This means that there is a way of picking some voters whose weights sum up to exactly $S$, and thus the sequence $s_{1}, \ldots, s_{n}$ can be partitioned into two subsequences that each sum up to $S$.

Our reduction can be carried out in polynomial time and so the proof is complete. This of course regards our default case, namely the nonunique case. The unique case also follows, namely, by observing that it is enough to add one voter with weight 1 and price 0 who votes for $p$. Then the same arguments as above show that this is a correct reduction.

The above theorems show that bribery is easy in the basic case but becomes intractable if we allow voters with prices and weights. It is natural to ask which of the additional features (prices? weights?) is responsible for making the problem difficult. It turns out that neither of them is the sole reason and that only their combination yields enough power to make the problem NP-complete. ${ }^{4}$

Theorem 3.3 Both plurality-\$bribery and plurality-weighted-bribery are in $\mathrm{P}$.

4. However, it is interesting to compare this to Theorems 4.8, 4.9, 4.13, and 4.15, which suggest that high weights are often the feature responsible for making the problem NP-complete. 
Theorem 3.3 is a special case of a result that we prove later (namely, of Theorem 3.8) and thus, instead of giving the proof, we provide a very informal discussion of polynomial-time algorithms for both plurality-\$bribery and plurality-weighted-bribery.

A direct greedy algorithm, like the one underpinning Theorem 3.1, fails to prove Theorem 3.3: The problem is that one has to judge whether it is better to bribe voters who currently prefer one of the winners or to bribe voters with the highest weights (or lowest prices). (To see that the former may sometime make sense, consider an election in which $a$ has two weight- 4 voters, $b$ has one weight- 5 voter, and $p$ has one weight- 2 voter. Bribing one weight- 4 voter is a winning bribery but bribing the one weight- 5 voter is not.)

We approach Theorem 3.3's proof as follows. Assume that $p$ will have $r$ votes after the bribery (or in the weighted case, vote weight $r$ ), where $r$ is some number to be specified later. If this is to make $p$ a winner, we need to make sure that everyone else gets at most $r$ votes. Thus we carefully choose enough cheapest (heaviest) voters of candidates that defeat $p$ so that after bribing them to vote for $p$ each candidate other than $p$ has at most $r$ votes. Then we simply have to make sure that $p$ gets at least $r$ votes by bribing the cheapest (the heaviest) of the remaining voters. If during this process $p$ ever becomes a winner without exceeding the budget (the bribe limit) then we know that bribery is possible.

How do we pick the value of $r$ ? In the case of plurality-\$bribery, we can simply run the above procedure for all possible values of $r$, i.e., $0 \leq r \leq\|V\|$, and accept exactly if it succeeds for at least one of them. For plurality-weighted-bribery a slightly trickier approach works. Namely, it is enough to try all values $r$ that can be obtained as a vote weight of some candidate (other than $p$ ) via bribing some number of his or her heaviest voters. There are only polynomially many such values and so the whole algorithm works in polynomial time. The intuition for using such values $r$ is the following: (a) When bribing voters of some candidate one can always limit oneself to the heaviest ones, and (b) after each successful bribery there is a value $r^{\prime}$ such that $p$ 's vote weight is at least $r^{\prime}$, each other candidate's vote weight is at most $r^{\prime}$, and there is some candidate $c \neq p$ such that $c^{\prime}$ s vote weight is exactly $r^{\prime}$. Our algorithm, in essence, performs an exhaustive search (within our heavily limited search space) for such a value $r^{\prime}$.

Note that all of the above algorithms assume that we bribe people to vote for $p$. This is a reasonable method of bribing if one wants $p$ to become a winner, but it also has potential real-world downsides: The more people we bribe, the more likely it may be that the malicious attempts will be detected and will work against $p$. To minimize the chances of that happening we might instead bribe voters to vote not for $p$ but for some other candidate(s). This way $p$ does not get extra votes but might be able to take away enough from the most popular candidates to become a winner.

Definition 3.4 plurality-weighted-negative-bribery is defined to be the same as pluralityweighted-bribery, except with the restriction that it is illegal to bribe people to vote for the designated candidate.

The problem plurality-negative-\$bribery is defined analogously. We call this setting negative-bribery because the motivation of $p$ is not to get votes for him- or herself, but to take them away from others. Unlike Theorem 3.3, this version of the problem draws a very sharp line between the complexity of bribing weighted and priced voters. 
Theorem 3.5 plurality-weighted-negative-bribery is NP-complete, but plurality-negative\$bribery is in $\mathrm{P}$.

Proof. We first give a polynomial-time algorithm for plurality-negative-\$bribery. Let $E=(C, V, p, k)$ be the bribery instance we want to solve. We need to make $p$ a winner by taking votes away from popular candidates and distributing them among the less popular ones. (The previous sentence said " $a$ winner" since we as usual are addressing the nonunique case. However, it is clear that a similar approach works for the unique case, i.e., the case in which the goal is to make $p$ "the winner.")

We partition the set of all candidates into three sets: candidates that defeat $p$, from whom votes need to be taken away, candidates that are defeated by $p$, to whom we can give extra votes, and candidates that have the same score as $p$. In the unweighted case, by score $_{E}(c)$ we mean the number of voters within $E$ who most prefer candidate $c$. In the weighted case, score $_{E}(c)$ means the total vote weight of voters within $E$ who most prefer $c$.

$$
\begin{aligned}
& C_{\text {above }}=\left\{c \mid c \in C, \text { score }_{E}(c)>\text { score }_{E}(p)\right\} . \\
& C_{\text {below }}=\left\{c \mid c \in C, \text { score }_{E}(c)<\text { score }_{E}(p)\right\} . \\
& C_{\text {equal }}=\left\{c \mid c \in C, \text { score }_{E}(c)=\text { score }_{E}(p)\right\} .
\end{aligned}
$$

Since all voters have the same weight (weight 1) in the current case, plurality-negative\$bribery, it is not hard to see that if there is some successful negative bribery then there will be some successful negative bribery that will bribe no voters into or out of $C_{\text {equal }}$ and that also won't bribe voters to move within their own "group," e.g., bribing a voter to shift from one $C_{\text {below }}$ candidate to another. (However, for the weights case, such "crazy" bribes are sometimes needed; see footnote 5.) To make sure that $p$ becomes a winner, for each candidate $c \in C_{\text {above }}$ we need to bribe as many of $c$ 's voters as are needed to reduce his or her score to at most $\operatorname{score}_{E}(p)$. Thus, altogether, we need to bribe $\sum_{c \in C_{\text {above }}}\left(\operatorname{score}_{E}(c)-\right.$ score $\left._{E}(p)\right)$ voters. The number of votes that a candidate $c \in C_{\text {below }}$ can accept without preventing $p$ from winning is $\sum_{c \in C_{\text {below }}}\left(\operatorname{score}_{E}(p)-\right.$ score $\left._{E}(c)\right)$. Thus, it is not hard to see that a negative bribery is possible exactly if the following inequality holds.

$$
\sum_{c \in C_{\text {above }}}\left(\operatorname{score}_{E}(c)-\text { score }_{E}(p)\right) \leq \sum_{c \in C_{\text {below }}}\left(\operatorname{score}_{E}(p)-\text { score }_{E}(c)\right) .
$$

If inequality (2) does not hold then we immediately reject. Otherwise, it remains to check whether the cost of our negative bribery is within the budget: For every candidate $c \in C_{\text {above }}$ let $b_{c}$ be the cost of bribing c's $\operatorname{score}_{E}(c)-\operatorname{score}_{E}(p)$ cheapest voters. If it holds that $\sum_{c \in C_{\text {above }}} b_{c} \leq k$ then we accept, as the negative bribery is possible. Otherwise we reject.

Clearly, our algorithm works in polynomial time. The correctness follows from the fact that we need to make all candidates in $C_{\text {above }}$ have score at most $\operatorname{score}_{E}(p)$ and for each $c \in C_{\text {above }} b_{c}$ is the lowest possible cost of achieving that. Equation (2) guarantees that the votes taken from candidates in $C_{\text {above }}$ can be distributed among those in $C_{\text {below }}$ without preventing $p$ from winning.

Now let us turn to showing the NP-hardness of plurality-weighted-negative-bribery. We must be careful here. In plurality-negative-\$bribery, we argued that one could without loss of generality ignore $C_{\text {equal }}$, i.e., one never needs to bribe voters into or out of $C_{\text {equal }}$, and that we can ignore bribing voters from one candidate in a group $\left(C_{\text {below }}, C_{\text {equal }}\right.$, and 
$C_{\text {above }}$ are our three groups) to another candidate within the same group. It is not too hard to see that that claim is false for the weights case, essentially due to the fact that, for example, members of $C_{\text {equal }}$ or $C_{\text {below }}$ can be useful in "making change" - that is, for splitting large weights into small ones. ${ }^{5}$ However, in the image of the reduction we are about to construct, $C_{\text {equal }}$ will contain only $p$, bribing votes to change to $p$ is forbidden by our "negative setting," and bribing votes to change away from $p$ clearly is never required for success; so we have a setting in which $C_{\text {equal }}$ in fact will not play any interesting role. And similarly, $\left\|C_{\text {above }}\right\|=\left\|C_{\text {below }}\right\|=1$ in the image of our reduction, so we will not have to worry about any within-a-group bribes.

Now, we start our construction to show the NP-hardness of plurality-weighted-negativebribery. In particular, we will construct a reduction from Partition. Let $s_{1}, \ldots, s_{n}$ be a sequence of nonnegative integers. We will design an instance of the pluralityweighted-negative-bribery such that bribery is possible if and only if $s_{1}, \ldots, s_{n}$ can be split into two parts that sum up to the same value. Let $S$ be such that $\sum_{i=1}^{n} s_{i}=2 S$. Our elections has three candidates: $p, c_{1}$, and $c_{2}$, and we have $n+1$ weighted voters:

1. $v_{0}$ with weight $S$, whose preferences are $p>c_{1}>c_{2}$, and

2. $v_{1}, \ldots, v_{n}$ with weights $s_{1}, \ldots, s_{n}$, each with preferences $c_{1}>c_{2}>p$.

The goal of the briber is to ensure $p$ 's victory via bribing up to $k=n+1$ voters (i.e., up to all the voters). Note that the only reasonable bribes are the ones that transfer votes of $v_{i}$, $1 \leq i \leq n$, from $c_{1}$ to $c_{2}$. (Strictly speaking, $v_{0}$ could legally be bribed to vote for $c_{1}$ or $c_{2}$, but that can be safely ignored.) If there is a set $A \subseteq\{1, \ldots, n\}$ such that

$$
\sum_{i \in A} s_{i}=S
$$

then we could bribe all voters $v_{i}, i \in A$, to vote for $c_{2}$ and all candidates would be winners. On the other hand, if $p$ can end up a winner by a bribery that does not ask anyone to vote for $p$, then there is a set $A$ that satisfies Equation (3): $p$ is a winner of our election if and only if each of $c_{1}$ and $c_{2}$ have vote weight exactly $S$. However, at the beginning $c_{1}$ holds $2 S$ vote weight and so a successful bribery needs to transfer exactly $S$ vote weight from $c_{1}$ to $c_{2}$. This is only possible if (3) holds for some $A$.

To finish the proof, we observe that this reduction can be computed in polynomial time.

Theorems 3.2 and 3.3 state that plurality-weighted-\$bribery is NP-complete but any attempt to make it simpler immediately pushes it back to the realm of $\mathrm{P}$. In fact, the

5. To see this, consider a setting where candidate Big is the most preferred candidate of one weight-10 voter and one weight- 2 voter, candidate $p$ is the most preferred candidate of one weight-10 voter, candidate MakeChange is the most preferred candidate of ten weight-1 voters, candidate SmallOne is the most preferred candidate of one weight-9 voter, candidate SmallTwo is the most preferred candidate of one weight-9 voter, and the limit on the number of bribes is $3 . C_{\text {above }}=\{B i g\}, C_{\text {equal }}=\{p$, MakeChange $\}$, and $C_{\text {below }}=\{$ SmallOne,SmallTwo $\}$. Note that there is no successful negative bribery that leaves MakeChange uninvolved. However, by moving from Big to MakeChange the weight-2 voter, and then by moving one weight-1 voter to each of SmallOne and SmallTwo from MakeChange, we have a successful negative bribery. This example uses $C_{\text {equal }}$ to make change, but one can construct similar examples that require one to bribe votes from one member of $C_{\text {below }}$ to another member of $C_{\text {below }}$. 
situation is even more dramatic. In the NP-complete problem plurality-weighted-\$bribery we assume that both prices and weights are encoded in binary. However, if either the prices or the weights are encoded in unary, then the problem, again, becomes easy. Before we proceed with a formal proof of this fact, let us discuss the issue in an informal manner. Why does the unary encoding of either one of the weights or the prices matter? The reason is that, for example, if the weights are encoded in unary then there trivially are only linearly many (with respect to the size of the input problem) different total weights of subsets of voters. Together with some additional tricks this allows us to use dynamic programming to obtain a solution.

Definition 3.6 plurality-weighted-\$briberyunary is defined exactly as is pluralityweighted-\$bribery, except the prices are to be encoded in unary. plurality-weighted unary\$bribery is plurality-weighted-\$bribery except with the weights encoded in unary.

It is tempting to use exactly the same proof approach as the one that we hinted at in the discussion below Theorem 3.3, i.e., to split the bribery into two parts: demoting others and promoting $p$. However, doing so would not be correct. Sometimes the optimal way of getting the scores of other candidates to be at most at a certain threshold $r$ prevents one from getting an optimal bribe for the complete problem. Consider an elections with three candidates, $c, d$, and $p$, and three voters $v_{1}, v_{2}$, and $v_{3}$ such that $v_{1}$ has both price and weight equal to $10, v_{2}$ has both price and weight equal to 7 , and $v_{3}$ has price 1,000,000 and weight 10. Both $v_{1}$ and $v_{2}$ prefer $c$ and $v_{3}$ prefers $d$. Clearly, an optimal bribe for the problem requires a threshold of 10 . The optimal way of getting $c$ down to vote weight at most 10 is by bribing $v_{2}$. However, at that point making $p$ a winner requires bribing $v_{1}$ as well. Yet, bribing just $v_{1}$ is a cheaper way of making $p$ a winner and getting $c$ below-or-equal-to the 10 threshold.

We will refer to plurality-weighted-\$briberyunary as the "unary prices case," and to plurality-weighted unary-\$bribery as the "unary weights case." We will now give an overview of how the algorithm works in the unary prices case, on input $E=(C, V, p, k)$. The unary weights case can be handled analogously. The main idea is that, using the fact that there are only linearly many possible prices to be paid, we can argue that there exists a polynomialtime computable function Heaviest $\left(E, C^{\prime}, \pi, r\right)$-where $C^{\prime}$ will be a subset of the candidates, $\pi$ will be an integer price, and $r$ will be an integer threshold - that gives the maximum vote weight that we can obtain by bribing voters of candidates in $C^{\prime}$ such that

1. the cost of this bribery is at most $\pi$,

2. after the bribery every candidate in $C^{\prime}$ has vote weight at most $r$.

To test whether it is possible to make $p$ a winner by spending at most $k$ dollars, we need to find a threshold $r$ such that $\operatorname{score}_{E}(p)+\operatorname{Heaviest}(E, C-\{p\}, k, r) \geq r$, i.e., so that the weight $p$ has originally or via bribed voters is at least as great as the post-bribery weight of each of the other candidates. Unfortunately, in the case of plurality-weighted\$briberyunary we cannot just try all thresholds since there may be exponentially many of them. Instead we use a strategy similar to the one that we hinted at when discussing Theorem 3.3. After every successful bribery (in elections with at least two candidates) there is some candidate $c \neq p$-namely, the candidate(s) other than $p$ with the greatest 
post-bribery total weight - that either is a tied-with- $p$ winner or loses only to $p$. We can use the after-bribery vote weight of this candidate to be the threshold for the bribery of the voters of all the other candidates. Of course, we neither know who this candidate is nor what vote weight he or she would have after a successful bribery. Nonetheless, we can try all candidates $c \neq p$ and for each such candidate and each possible "sub-budget" $b \leq k$ can ask what is the maximum amount of additional weight we can get for $p$ from bribing $c$ 's voters when allowed to spend at most $b$ to do so (this will require solving certain instances of the knapsack problem). Then, using the thus obtained threshold, we can bribe the voters of the rest of the candidates. There are (at most) linearly many candidates and (at most) linearly many prices so this yields (at most) polynomially many combinations.

Let us now describe how the above plan can be implemented. We no longer limit ourselves to the unary prices case, but describe both cases in parallel. Let $E=(C, V, p, k)$ be our input. For each candidate $c \in C$ we define

$$
V_{E}^{c}=\{v \in V \mid c \text { is the most preferred candidate of } v\} .
$$

Since we do not have any additional restrictions it only makes sense to bribe voters to support $p$. For a given candidate $c \in C$, we can describe our bribing options either as a function that gives the highest weight of $c$ 's voters we can bribe for $b$ dollars or as a function that gives the lowest price needed to gain vote weight at least $w$ by bribing $c$ 's voters.

$$
\begin{aligned}
\text { heaviest }(E, c, b) & =\max \left\{\omega(U) \mid U \subseteq V_{E}^{c} \text { and } \pi(U) \leq b\right\} \\
\text { cheapest }(E, c, w) & =\min \left\{\pi(U) \mid U \subseteq V_{E}^{c} \text { and } \omega(U) \geq w\right\}
\end{aligned}
$$

If $c$ is not a candidate in $E$, these functions are undefined. Here and in the rest of the proof, we take the max and min of the empty set to be undefined. Note that if $c$ is a candidate in $E$, then heaviest $(E, c, b)$ is defined for all $b \geq 0$ and cheapest $(E, c, w)$ is defined for all $w \leq \omega\left(V_{E}^{c}\right)$. Also note that heaviest can easily be computed in polynomial time in the unary prices case and that cheapest can easily be computed in polynomial time in the unary weights case. In both cases we simply use dynamic programming solutions for an appropriate optimization variant of the knapsack problem. ${ }^{6}$ We can further generalize these functions to give us information about the best bribes regarding sets of candidates. For each $U \subseteq V$, we define $\operatorname{bribed}(E, U)$ to be the bribery problem exactly like $E$ but with the voters in $U$ bribed to vote for $p$. We define

$$
\begin{aligned}
& \text { Heaviest }\left(E, C^{\prime}, b, r\right)=\max \left\{\begin{array}{l|l}
\omega(U) \mid \begin{array}{l}
\left(U \subseteq \bigcup_{c \in C^{\prime}} V_{E}^{c}\right) \wedge(\pi(U) \leq b) \wedge \\
\left(\forall c \in C^{\prime}\right)\left[\operatorname{score}_{\text {bribed }(E, U)}(c) \leq r\right]
\end{array}
\end{array}\right\}, \text { and } \\
& \text { Cheapest }\left(E, C^{\prime}, w, r\right)=\min \left\{\begin{array}{l|l}
\pi(U) \mid \begin{array}{l}
\left(U \subseteq \bigcup_{c \in C^{\prime}} V_{E}^{c}\right) \wedge(\omega(U) \geq w) \wedge \\
\left(\forall c \in C^{\prime}\right)\left[\operatorname{score}_{\text {bribed }(E, U)}(c) \leq r\right]
\end{array}
\end{array}\right\}
\end{aligned}
$$

If $C^{\prime}$ is not a subset of $E$ 's candidate set, these functions are undefined.

6. The knapsack problem is the following. Given a set of items, each with a price $\pi$ and a weight $\omega$, is it possible to select items with total weight at least $W$, but without exceeding total price $K$ ? It is well known that the knapsack problem has a polynomial-time dynamic programming algorithm if either the prices are encoded in unary or the weights are encoded in unary. (See the work of Martello \& Toth, 1990, for background/reference on the knapsack problem.) 
Lemma 3.7 We consider now only elections in which each voter has both a price and a weight. If prices are encoded in unary then there is an algorithm that computes Heaviest in polynomial time. If weights are encoded in unary then there is an algorithm that computes Cheapest in polynomial time.

Proof. Note that in the unary prices case there are only linearly many sub-budgets $b$ for which we need to compute the value of Heaviest, namely $0 \leq b \leq \pi(V)$, and in the unary weights case there are only linearly many weights $w$ for which we need to evaluate Cheapest, namely $0 \leq w \leq \omega(V)$. Using this fact we provide dynamic programming algorithms for computing both functions. For the base case we have the following: If $c$ is not a candidate of $E$, then both our functions are undefined. Otherwise,

$$
\begin{aligned}
\text { Heaviest }(E,\{c\}, b, r) & =\left\{\begin{array}{l}
\operatorname{leaviest}(E, c, b) \\
\text { if } \text { score }_{E}(c)-\text { heaviest }(E, c, b) \leq r, \\
\text { otherwise. }
\end{array}\right. \\
\text { Cheapest }(E,\{c\}, w, r) & = \begin{cases}\operatorname{cheapest}(E, c, w) & \text { if } \operatorname{score}_{E}(c)-w \leq r, \\
\operatorname{cheapest}\left(E, c, \text { score }_{E}(c)-r\right) & \text { otherwise }\end{cases}
\end{aligned}
$$

The following observation allows us to compute Cheapest and Heaviest for larger sets. We assume that $C^{\prime}$ does not contain $c$. If any of the candidates in $C^{\prime} \cup\{c\}$ are not candidates of $E$, then both our functions are undefined. Otherwise,

$$
\begin{aligned}
& \text { Heaviest }\left(E, C^{\prime} \cup\{c\}, b, r\right)=\max \left\{\text { Heaviest }\left(E, C^{\prime}, b^{\prime}, r\right)+\text { Heaviest }\left(E,\{c\}, b-b^{\prime}, r\right) \mid\right. \\
& \left.0 \leq b^{\prime} \leq b \text { and Heaviest }\left(E, C^{\prime}, b^{\prime}, r\right) \text { and Heaviest }\left(E,\{c\}, b-b^{\prime}, r\right) \text { are both defined }\right\} . \\
& \text { Cheapest }\left(E, C^{\prime} \cup\{c\}, w, r\right)=\min \left\{\text { Cheapest }\left(E, C^{\prime}, w^{\prime}, r\right)+C h e a p e s t\left(E,\{c\}, w-w^{\prime}, r\right) \mid\right. \\
& \left.0 \leq w^{\prime} \leq w \text { and Cheapest }\left(E, C^{\prime}, w^{\prime}, r\right) \text { and Cheapest }\left(E,\{c\}, w-w^{\prime}, r\right) \text { are both defined }\right\} .
\end{aligned}
$$

Thus, in the unary prices case we can compute Heaviest $\left(E, C^{\prime}, b, r\right)$ using dynamic programming in polynomial time. The same applies to Cheapest $\left(E, C^{\prime}, w, r\right)$ for the unary weights case.

Theorem 3.8 Both plurality-weighted-\$briberyunary and plurality-weighted unary- $^{-}$ \$bribery are in $\mathrm{P}$.

Proof. Algorithms for both of the problems are very similar and we will describe only the (nonunique) unary prices case in detail. We provide the pseudocode for the (nonunique) unary weights case, but we omit its proof of correctness, which is analogous to the proof for the unary prices case. We mention in passing that the two unique cases can easily be obtained as well, via the natural modifications of our algorithm.

Figure 1 shows our procedure for the unary prices case. The idea of the algorithm is the following: Suppose that there is a set $B$ of voters such that if we bribe all members of $B$ to vote for $p$ then $p$ becomes a winner. We can assume that for each candidate $c$, $c$ 's voters have been bribed optimally, i.e., there is no cheaper way of getting the same (or greater) vote weight by bribing a different subset of $c$ 's voters. There is some candidate $c$ that has the most votes among the non- $p$ candidates after bribery. Thus, to decide if bribery is possible it is enough to test whether there is a candidate $c \neq p$ and a sub-budget $b, 0 \leq b \leq k$, such that after bribing $c$ 's voters optimally, spending $b$ dollars, it is still possible to bribe (without, overall, exceeding the budget) voters of the other candidates in such a way that 


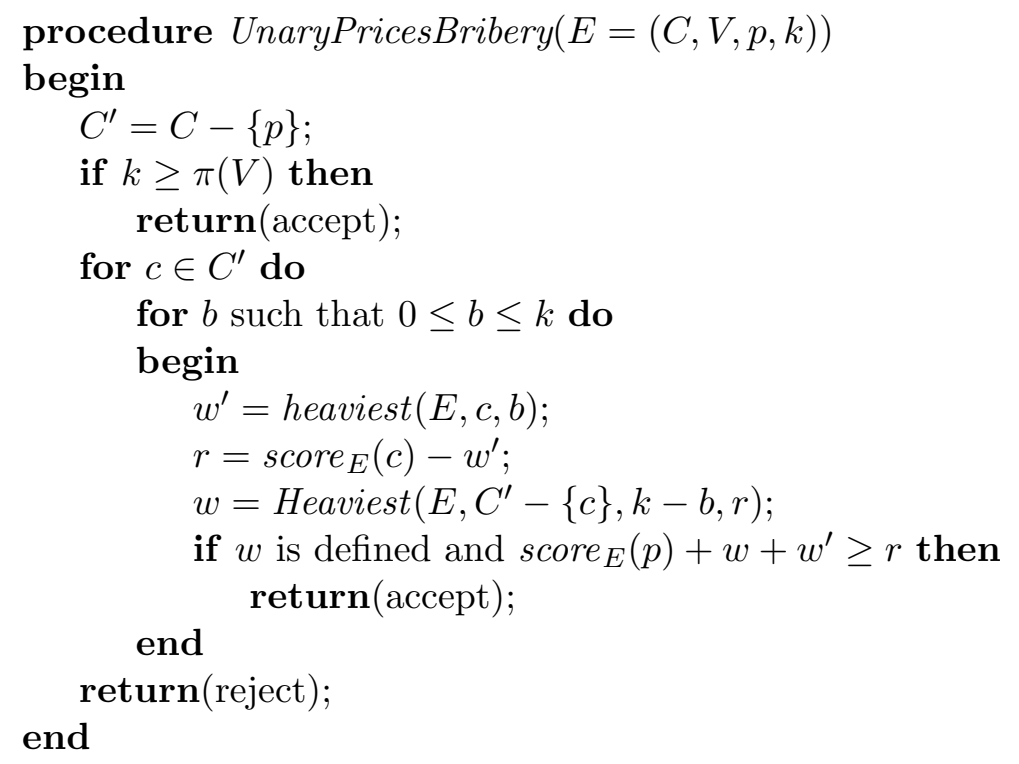

Figure 1: The main procedure for plurality-weighted-\$briberyunary.

1. each candidate ends up with vote weight not higher than that of $c$, and

2. enough voters can be bribed so that $p$ becomes a winner.

Our algorithm tests exactly if this is the case and accepts if so. (Though its "if-then" line might at first seem to focus just on having the candidates in $C-\{c\}$ beat $p$, note that $c$ 's post-bribery score is $r$, so that line handles $c$ also.) By the above reasoning, if bribery is possible the algorithm accepts. It should also be clear that if the algorithm accepts then bribery is indeed possible. Since the functions heaviest and Heaviest can be computed in polynomial time, we have that the whole algorithm runs in polynomial time. Thus, plurality-weighted-\$briberyunary is in $\mathrm{P}$.

An analogous algorithm works for the unary weights case, see Figure 2. The proof of correctness is analogous to the unary prices case.

Theorem 3.8 is particularly interesting because it says that plurality-weighted-\$bribery will be difficult only if we choose both weights and bribe prices to be high. However, the prices are set by the voters, and in many cases one could assume that they would set them to be fairly low, in some sense rendering the bribery problem easy.

Another possible attack on the complexity of plurality-weighted-\$bribery is through approximation algorithms. In fact, using Theorem 3.8, Faliszewski (2008) obtained a fullypolynomial approximation scheme for plurality-weighted-\$bribery. Many researchers ask about typical-case complexity of practically encountered NP-complete problems (see the work of Conitzer \& Sandholm, 2006; Procaccia \& Rosenschein, 2007; Erdélyi, Hemaspaandra, Rothe, \& Spakowski, 2007, for discussions of this issue in the context of voting problems; see also Erdélyi, Hemaspaandra, Rothe, \& Spakowski, to appear; Erdélyi, Hemaspaandra, 


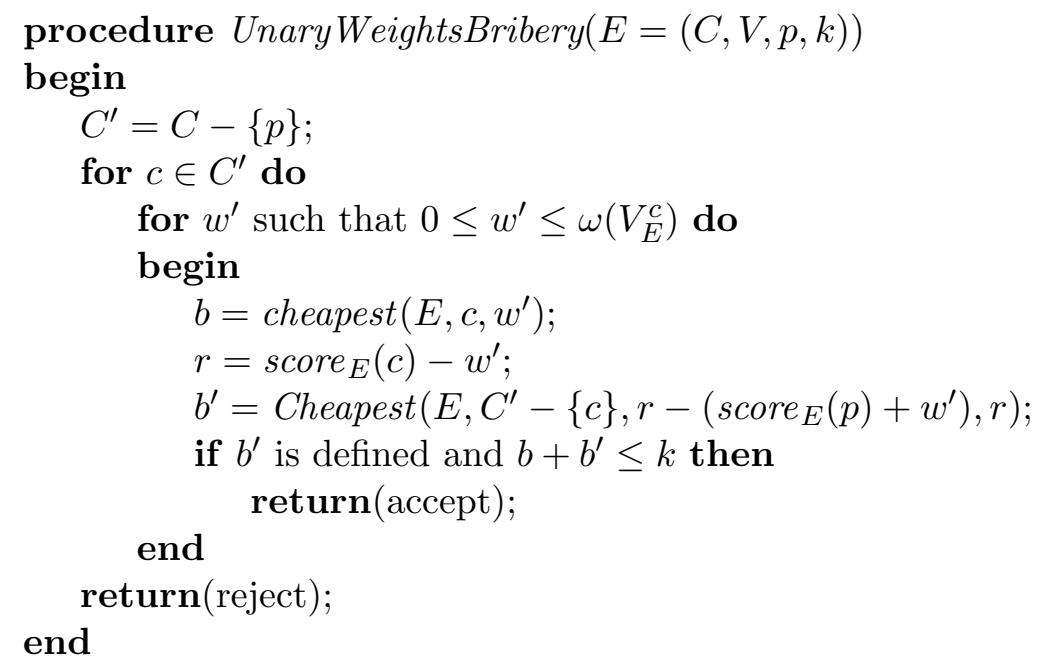

Figure 2: The main procedure for plurality-weighted $u n a r y-\$ b r i b e r y$.

Rothe, \& Spakowski, 2009), and this is clearly an important direction. ${ }^{7}$ However, it is often difficult to come up with a distribution of inputs that is both realistic and simple enough to study. On the other hand, providing a good polynomial-time approximation algorithm would be a worst-case result: No matter how difficult an instance we would be given, we could compute a decent answer. Recent papers by Brelsford et al. (2008), Faliszewski (2008), and Zuckerman et al. (2008) take steps in this interesting direction.

7. There has been much excitement over the recent paper "Elections Can Be Manipulated Often" (Friedgut, Kalai, \& Nisan, 2008, see also Dobzinski \& Procaccia, 2008; Xia \& Conitzer, 2008), and indeed a referee commented to us that that paper proves that (under certain assumptions) elections are manipulable "most of the time." However, one in fact has to be a bit careful as to what one claims. The lower bound that paper (given its assumptions) establishes for frequency of manipulation is, for a type of problem that is related to unpriced bribery (but involves choosing a single manipulator after drawing the votes from one's distribution), $\Omega(1 /\|V\|)$, and for the more typical approach to manipulation of focusing on a single voter, that itself gives a lower bound on frequency of manipulation of $\Omega\left(1 /\|V\|^{2}\right)$. That isn't "most," of the time, but rather goes to zero asymptotically. Even if that lower bound could be tremendously raised to $\Omega(1)$, that still might mean a manipulation frequency of 0.00001 percent of the time - not a frequency that in itself means election methods are very frequently open to manipulation. This is an exciting, active research direction in which we suspect that future work will much clarify the upper and lower bounds that hold on frequency of manipulation (both in the single-manipulator case and the coalition manipulation case), and what assumptions about election systems are needed to obtain such results. Changing topics, we mention that in some cases "most of the time" detours around NP-hardness election-complexity results have already been obtained. For example, although the winner problem in Dodgson elections is known to be complete for parallel access to NP, two recent papers, by McCabe-Dansted, Pritchard, and Slinko (2008) and by Homan and Hemaspaandra (2009), have shown that (under particular assumptions about distributions and the relationship between the numbers of candidates and voters) there are heuristic algorithms that in a rigorous sense are correct most of the time. 


\section{Bribery Versus Manipulation, and Two Dichotomy Theorems}

The previous section provided a detailed discussion of the complexity of bribery for plurality voting. To obtain its results we carefully hand-crafted and altered various algorithms and reductions. Designing algorithms and reductions for specific bribery problems is certainly a reasonable approach, but even better would be to find more general tools for establishing the complexity of bribery in elections. Such general tools would be especially interesting if they allowed one to inherit results already existent in the literature on election systems. In this section we implement the above plan by studying relationships between bribery and manipulation, and by showing how to obtain results using the relationships we find. In the next section, by studying some ways in which integer programming can be employed to solve bribery problems we continue this emphasis on exploring flexible tools for establishing the complexity of bribery. There, using a theorem of Lenstra we show many bribery problems regarding elections with fixed-size candidate sets to be in $\mathrm{P}$, even when the voters are succinctly represented. (Regarding the coming results studying the relationship between bribery and manipulation, we more generally commend to the reader the issue of finding even more extensive links between such problems as bribery, manipulation, and control. We find this a natural and important direction.)

Manipulation is in flavor somewhat similar to bribery, with the difference that in manipulation the set of voters who may change their preference lists is specified by the input. Formally, if $\mathcal{E}$ is some election rule then $\mathcal{E}$-manipulation is the following problem (e.g., see Bartholdi et al., 1989a; Conitzer et al., 2007).

Name: $\mathcal{E}$-manipulation.

Given: A set $C$ of candidates, a collection $V$ of voters specified via preference lists, a set $S$ of manipulative voters (without loss of generality, not including any members of $V$ ), and a candidate $p \in C$.

Question: Is there a way to set the preference lists of the voters in $S$ so that under election rule $\mathcal{E}$ the voters in $S \cup V$ together choose $p$ as a winner?

Instances of the manipulation problems can be described as tuples $(C, V, S, p)$, where $C$ is a list of candidates, $V$ is a list of voters (in the same format as in the bribery problems), $S$ is a list of the manipulative voters, and $p$ is the designated candidate that voters in $S$ want to be a winner (a unique winner, in the unique case).

Manipulation, just like bribery, comes in many flavors. We may be asked to make $p$ the unique winner or just a winner, voters may have weights (in which case $S$ is specified together with weights of voters in $S$ ), etc. Bribery can be viewed as manipulation where the set of manipulators is not fixed in advance and where deciding who to manipulate is a part of the challenge. Note that to check whether bribery can be successful on a given input we can simply try all possible manipulations by $k$ voters, where $k$ is the number of bribes we are willing to allow. In this way, for a fixed $k$, we can disjunctively truth-table reduce any bribery problem to the analogous manipulation problem. (Note that having no prices but a limit of bribing $k$ voters is the same in effect as having unit prices and a budget of $k$.)

Theorem 4.1 Let $k$ be an arbitrary positive integer. Let $\mathcal{B}$ be any of our bribery problems, but with the following constraints: Voters have no prices (i.e., we do not consider \$bribery 
problems) and bribing more than $k$ voters is forbidden (that is, we require that in each "yes"-instance $\left(C, V, p, k^{\prime}\right)$ of $\mathcal{B}$ we have $\left.k^{\prime} \leq k\right)$. Let $\mathcal{M}$ be the analogous manipulation problem, i.e., the manipulation problem for the same election system, with weighted voters if $\mathcal{B}$ allows that, allowing the manipulating set to contain any number of voters between 0 and $k$. Then it holds that $\mathcal{B} \leq_{d t t}^{p} \mathcal{M}$.

Proof. To show that $\mathcal{B} \leq_{d t t}^{p} \mathcal{M}$ we need to give a polynomial-time procedure that for an input $x$ outputs a list of strings $y_{1}, \ldots, y_{m}$ such that $x \in \mathcal{B}$ if and only if at least one of $y_{i}$, $1 \leq i \leq m$, is in $\mathcal{M}$. We now describe such a procedure.

Let $x$ be the input string. We first check whether $x$ can be parsed as an instance of $\mathcal{B}$ (reminder: that is, that $x$ meets the syntactic constraints of $\mathcal{B}$ ). If not then we output an empty list and terminate; otherwise we decode $V$, the voter set, and $k^{\prime} \leq k$, the maximum number of voters that can be bribed, from the string $x$. For every subset $W$ of $k^{\prime \prime}=\min \left(k^{\prime},\|V\|\right)$ elements of $V$ we form an instance of the manipulation problem with voter set $V-W$ and manipulating set equal to $W$. After we go through all $k^{\prime \prime}$-element subsets we output the list of all the manipulation instances that we formed.

This procedure clearly works in polynomial time as there are at most $\left(\begin{array}{c}\|V\| \\ k\end{array}\right)=O\left(\|V\|^{k}\right)$ sets to test and we can form instances of manipulation in polynomial time. If any of the manipulation instances we output is in $\mathcal{M}$ then bribery is possible; it is enough to bribe exactly the voters selected for the manipulating group. On the other hand, if bribery is possible, then at least one of the instances we output belongs to $\mathcal{M}$, namely any one that includes the voters we would bribe.

While simple, this result is still powerful enough to allow the inheritance of some results from previous papers. Bartholdi et al. (1989a) discuss manipulation by single voters and Theorem 4.1 translates their results to the bribery case. In particular, this translation says that bribery for $k=1$ is in $\mathrm{P}$ for plurality, Borda count, and many other systems.

Can we strengthen Theorem 4.1 from constant-bounded bribery to general bribery? The answer is no: There are election systems for which bribery is NP-complete but manipulation is in P. In particular, manipulation for approval voting (both in the weighted and the unweighted case) is in $\mathrm{P}$ for any size of manipulating set: The manipulating group simply approves just of their favorite candidate and nobody else. ${ }^{8}$ However, by the following theorem, bribery for approval voting is NP-complete.

Theorem 4.2 approval-bribery is NP-complete.

Proof. Clearly, approval-bribery is in NP. NP-completeness follows from a reduction from X3C.

Let $B=\left\{b_{1}, \ldots, b_{3 t}\right\}$ and let $S=\left\{S_{1}, \ldots, S_{m}\right\}$ be a family of three-element subsets of $B$. Without loss of generality, we assume that $m \geq t$; otherwise an exact cover is impossible. For each $i, 1 \leq i \leq 3 t$, let $\ell_{i}$ be the number of sets $S_{j}$ that contain $b_{i}$. On input $(B, S)$ we construct approval-bribery instance $E=(C, V, p, k)$, where $k=t$, the set of candidates $C$ is equal to $B \cup\{p\}$, and we have the following voters.

1. For each $S_{i} \in S$ there is a voter $v_{i}$ who approves of exactly the members of $S_{i}$.

8. Meir et al. (2008) in a somewhat different and more flexible setting have previously noted that approvalmanipulation is in $\mathrm{P}$ if there is only one manipulator. 
2. For each $b_{i}$ we have $m-\ell_{i}+1$ voters who approve only of $b_{i}$.

3. We have $m-t$ voters who approve only of $p$.

Note that $p$ gets $m-t$ approvals and that each $b_{i}, 1 \leq i \leq 3 t$, gets $m+1$ approvals. We claim that $p$ can be made a winner by bribing at most $t$ voters if and only if $B$ has an exact cover by sets in $S$.

First assume that there is a set $A$ such that $\|A\|=t$ and $\bigcup_{i \in A} S_{i}=B$. To make $p$ a winner, bribe each $v_{i}$ such that $i \in A$ to approve only of $p$. As a result $p$ gets $m$ approvals and each $b_{i}$ loses exactly one approval. Thus, all candidates are winners. On the other hand, assume there is a bribery of at most $t$ voters that makes $p$ a winner. Each bribed voter contributes at most one additional approval for $p$. Thus, $p$ will get at most $m$ approvals. Each candidate in $B$ has $m+1$ approvals, and our bribery needs to take away at least one approval from each candidate in $B$. Since we bribe at most $t$ voters, this can only happen if we bribe $t$ voters $v_{i}$ that correspond to a cover of $B$.

This reduction can be computed in polynomial time.

Of course, when the number of bribes is bounded by some fixed constant then, by Theorem 4.1, approval-bribery can be solved in polynomial time.

We mention that bribery in approval elections is actually very easy if we look at a slightly different model. Our bribery problems allow us to completely modify the approval vector of a voter, but this may be too demanding. A voter might be willing to change some of his or her approval vector's entries but not to change it completely.

Definition 4.3 approval-bribery' is the problem that takes as input a description of an approval election along with a designated candidate $p$ and a nonnegative integer $k$, and asks whether it is possible to make $p$ a winner by at most $k$ entry changes (total) in the approval vectors. approval-\$bribery' is defined analogously, but with the extra twist that now changing each entry of an approval vector may have a different price. ${ }^{9}$

Having different prices for flipping different entries in approval-\$bribery' models the possibility that a voter might be more willing to change his or her approval of some candidates than of other candidates. These modified problems turn out to be easy. In fact, they are easy even if we have both weights and prices, provided one of them is encoded in unary.

Theorem 4.4 Both approval-weighted-\$bribery'unary and approval-weightedunary\$bribery' are in $\mathrm{P}$.

Proof. The polynomial-time algorithm we provide is based on the observation that in both approval-weighted-\$bribery ${ }_{\text {unary }}$ and approval-weighted unary-\$bribery $^{\prime}$ getting vote weight for the favorite candidate can be (carefully) treated separately from demoting the other candidates. (This is basically because in approval voting in the bribery' model costs are linked to entries in voters' approval vectors and a candidate's point total is by weighted addition, over all the voters, of that candidate's 0-or-1 entry from that voter.)

9. As a referee points out, from a technical perspective one can view the voters in approval-bribery' (and its variants) as broken into multiple "plurality voters that can be turned on and off." Partially due to this similarity, various flavors of approval-bribery' have computational properties very similar to the corresponding variants of plurality-bribery. 
We can divide any bribery into two phases: First, we bribe voters to approve of $p$, our favorite candidate, and second, we bribe enough voters to retract their approvals of candidates that still defeat $p$. There are only polynomially many relevant vote weights that $p$ may obtain by bribery, so we can try them all.

Let $E=(C, V, p, k)$ be the bribery instance we need to solve. For a candidate $c$, a price $b$, and a subset of voters $V^{\prime}$, we define heaviest $\left(V^{\prime}, c, b\right)$ to be the highest vote weight of voters in $V^{\prime}$ whose approval of $c$ can be switched by spending at most $b$ dollars. Similarly, for a candidate $c$, vote weight $w$, and a subset of voters $V^{\prime}$, we define cheapest $\left(V^{\prime}, c, w\right)$ to be the lowest price that can switch the approval-of-c of voters in $V^{\prime}$ that have total weight at least $w$. In our proof we only use sets $V^{\prime}$ where either all voters approve of $c$ or all voters disapprove of $c$. Note that heaviest $\left(V^{\prime}, c, b\right)$ is defined for all $b \geq 0$ and that cheapest $\left(V^{\prime}, c, w\right)$ is defined for all $w \leq \omega\left(V^{\prime}\right)$. As in Section 3, heaviest can easily be computed in polynomial time in the unary prices case and cheapest can easily be computed in polynomial time in the unary weights case. In addition, cheapest can be computed in polynomial time in the unary prices case. Note that

$$
\operatorname{cheapest}\left(V^{\prime}, c, w\right)=\min \left\{b \mid \text { heaviest }\left(V^{\prime}, c, b\right) \geq w\right\} .
$$

Since there are only polynomially many prices to try, this can be done in polynomial time.

Figure 3 gives pseudocode for the procedure UnaryPricesApproval, which decides approval-weighted-\$briberyunary' ${ }^{\prime}$ score $_{E}(c)$ denotes the number of approvals of candidate $c$ in election $E$. The procedure simply tries all relevant weights that $p$ could obtain by bribery and tests whether it is possible, for any of them, to bring the other candidates down to vote weight at most that of $p$ without exceeding the budget. The procedure is correct because of the separation we achieved (as discussed above, and applied within our proof framework of trying all thresholds) between the issue of bribing voters to approve of $p$ and the issue of bribing them not to approve of some other candidate. Also, as cheapest and heaviest are computable in polynomial time, the procedure works in polynomial time. An analogous procedure decides the unary weights case: Simply change the line "for $b=0$ to $k$ do" to "for $w=0$ to $\omega\left(V^{\prime}\right)$ do" and the line " $w=$ heaviest $\left(V^{\prime}, p, b\right)$ " to " $b=\operatorname{cheapest}\left(V^{\prime}, p, w\right) . "$

With both prices and weights encoded in binary, approval-weighted-\$bribery ${ }^{\prime}$ becomes NP-complete.

Theorem 4.5 approval-weighted-\$bribery' is NP-complete.

Proof. It is immediate that approval-weighted-\$bribery ${ }^{\prime}$ is in NP. To show NP-hardness, we will construct a reduction from Partition. Let $s_{1}, \ldots, s_{n}$ be a sequence of nonnegative integers and let $\sum_{i=1}^{n} s_{i}=2 S$. We construct an election $E$ with candidates $p$ and $c$ and $n+1$ voters, $v_{0}, \ldots, v_{n}$, with the following properties.

1. $v_{0}$ has weight $S$, approves only of $p$, and changing any of $v_{0}$ 's approvals costs $2 S+1$.

2. $v_{i}$, for $1 \leq i \leq n$, has weight $s_{i}$, approves only of $c$, changing $v_{i}$ 's approval for $p$ costs $s_{i}$, and changing $v_{i}$ 's approval for $c$ costs $2 S+1$.

We claim that $p$ can be made a winner by a bribery of cost at most $S$ if and only if there is a set $A \subseteq\{1, \ldots, n\}$ such that $\sum_{i \in A} s_{i}=S$. 


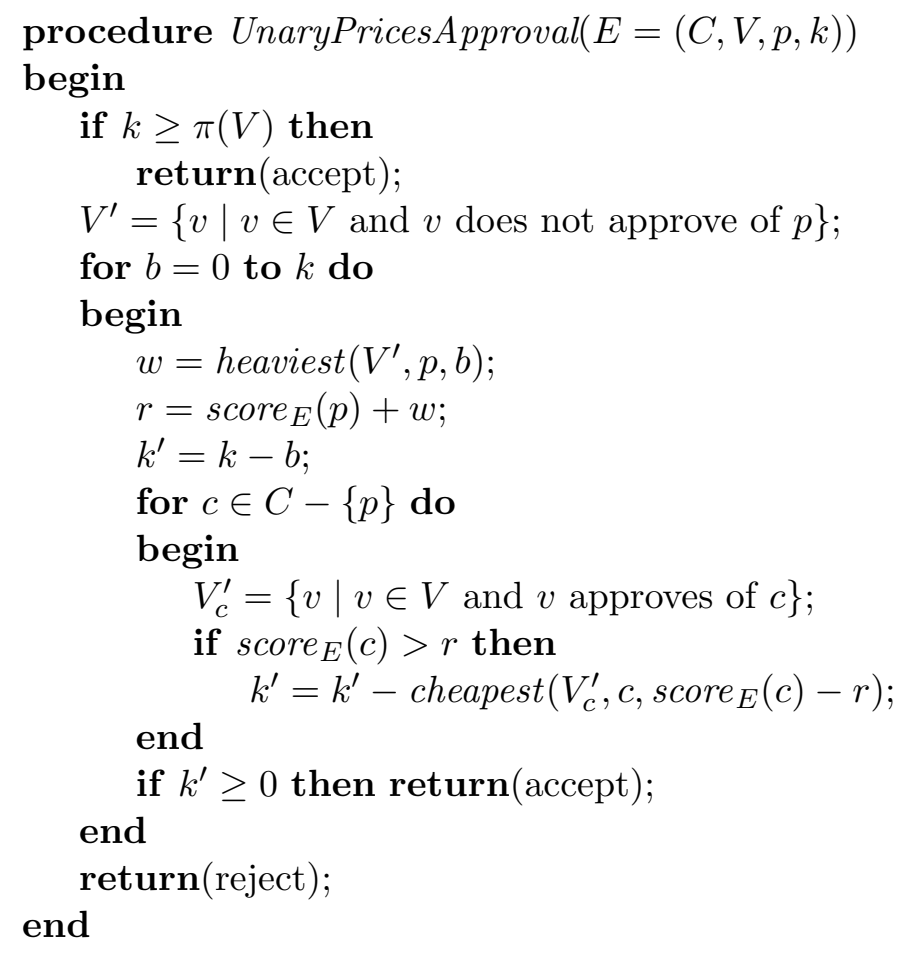

Figure 3: The procedure UnaryPricesApproval.

First suppose that $p$ can be made a winner by a bribery of cost at most $S$. Then we can only bribe voters $v_{1}, \ldots, v_{n}$ to approve of $p$. In election $E, p$ has $S$ approvals and $c$ has $2 S$ approvals, so our bribery needs to give $p$ at least $S$ extra approvals. Since changing $v_{i}$ 's approval of $p$ costs $s_{i}$, and the weight of $v_{i}$ is also $s_{i}$, it follows that $p$ gains exactly $S$ approvals, and that the weights of the bribed voters in $v_{1}, \ldots, v_{n}$ add up to exactly $S$. This implies that the sequence $s_{1}, \ldots, s_{n}$ can be partitioned into two subsequences that each sum to $S$.

On the other hand, assume there is a set $A \subseteq\{1, \ldots, n\}$ such that $\sum_{i \in A} s_{i}=S$. Then we can bribe voters $v_{i}, i \in A$, to approve of $p$. As a result, both $p$ and $c$ will have vote weight $2 S$ and both of them will be winners. Our reduction can be computed in polynomial time and thus the theorem is proved.

Which of the above-discussed bribery models for approval is more appropriate depends on the setting. For example, bribery' seems more natural when we look at the web and treat web pages as voting by linking to other pages. It certainly is easier to ask a webmaster to add/remove a link than to completely redesign the page. We point the reader to the work of Faliszewski (2008) for further discussion of bribery scenarios similar to bribery'.

After this somewhat lengthy discussion of approval bribery, let us now return to our central goal of relating bribery and manipulation. In Theorem 4.1 we managed to disjunctively truth-table reduce a restricted version of bribery to manipulation. The discussion and theorems that follow show that working in the opposite direction, reducing manipulation to bribery, which at first might seem more difficult, is in fact more fruitful. 
The reason why reducing manipulation to bribery appears to be more difficult is that bribery allows more freedom to the person interested in affecting the elections. To embed manipulation within bribery, we have to find some way of expressing the fact that only a certain group of voters should be bribed (or, at least, expressing the fact that if there is any successful bribery then there is also one that only bribes the manipulators). We can fairly easily implement this plan, though at the cost of reducing to a stronger bribery model, namely bribery with prices.

Theorem 4.6 Let $\mathcal{M}$ be some manipulation problem and let $\mathcal{B}$ be the analogous $\$$ bribery problem (for the same election system). It holds that $\mathcal{M} \leq_{m}^{p} \mathcal{B}$.

Proof. Let $M=(C, V, S, p)$ be an instance of $\mathcal{M}$. We design an instance $B$ of $\mathcal{B}$ such that $B=\left(C, V^{\prime} \cup S^{\prime}, p, 0\right)$, where

1. $V^{\prime}$ is equal to $V$, except that each voter has price 1 , and

2. $S^{\prime}$ is equal to $S$, except that each voter has price 0 and some fixed arbitrary preference list.

Since the bribery budget is set to zero, the only voters that we may possibly bribe are those in $S^{\prime}$. The preference lists of the voters in $S^{\prime}$ after any such bribery directly correspond to a manipulation in $M$. This reduction can be carried out in polynomial time.

Clearly, Theorem 4.6 holds even for \$bribery problems where prices are represented in unary or are required to come from the set $\{0,1\}$. Theorem 4.6 is very useful as it allows us to inherit some very powerful results from the theory of manipulation. Hemaspaandra and Hemaspaandra proved the following dichotomy result (see also Procaccia \& Rosenschein, 2007; Conitzer et al., 2007).

Theorem 4.7 (Hemaspaandra \& Hemaspaandra, 2007) Let $\alpha=\left(\alpha_{1}, \ldots, \alpha_{m}\right)$ be a scoring protocol. If it is not the case that $\alpha_{2}=\alpha_{3}=\cdots=\alpha_{m}$, then $\alpha$-weightedmanipulation is NP-complete; otherwise, it is in $\mathrm{P}$. This result holds for both the unique and nonunique variants.

Combining the two above theorems with Theorem 3.2 we can immediately classify the complexity of weighted-\$bribery for all scoring protocols.

Theorem 4.8 For each scoring protocol $\alpha=\left(\alpha_{1}, \ldots, \alpha_{m}\right)$, if $\alpha_{1}=\alpha_{m}$ then $\alpha$-weighted\$bribery is in $\mathrm{P}$; otherwise it is $\mathrm{NP}$-complete.

Proof. We consider three cases.

1. $\alpha_{1}=\cdots=\alpha_{m}$.

2. $\alpha_{1}>\alpha_{2}=\cdots=\alpha_{m}$.

3. All other settings.

In the first case, $\alpha_{1}=\cdots=\alpha_{m}, \alpha$-weighted-\$bribery is trivially in $\mathrm{P}$ as all candidates are always tied. For the remaining two cases, note that $\alpha$-weighted-\$bribery is clearly in NP. It remains to show NP-hardness. 
In the second case, $\alpha_{1}>\alpha_{2}=\cdots=\alpha_{m}$, we can employ the proof of Theorem 3.2. Theorem 3.2 shows NP-hardness for $(1,0)$-weighted-\$bribery. It is easy to see that for all $m \geq 2$ we can pad this reduction with $m-2$ candidates that are never ranked first to obtain NP-hardness for $(1, \overbrace{0, \ldots, 0}^{m-1})$-weighted-\$bribery. Note that our $\alpha$ describes elections equivalent to plurality (i.e., a candidate is a winner of an $\alpha$ election if and only if he or she would also be a winner of the $(1, \overbrace{0, \ldots, 0}^{m-1})$ election with the same voters and candidates; see Observation 2.2 in the paper of Hemaspaandra \& Hemaspaandra, 2007). Thus, we get NP-completeness of $\alpha$-weighted-\$bribery for this case since we do have at least two candidates.

The third case follows by combining Theorem 4.6 with Theorem 4.7. Since $\alpha$-weightedmanipulation many-one reduces to $\alpha$-weighted-\$bribery and $\alpha$-weighted-manipulation is NP-complete we have that $\alpha$-weighted-\$bribery is NP-hard. This exhausts all cases.

Theorem 4.8 applies to $\$$ bribery, but of course it is also interesting to ask what happens in the case when voters do not have prices. Does bribery remain NP-complete? Can we express the constraints of bribery without using such a direct embedding as above? The following dichotomy theorem shows that the answer is "Yes, but in fewer cases."

Theorem 4.9 For each scoring protocol $\alpha=\left(\alpha_{1}, \alpha_{2}, \ldots, \alpha_{m}\right)$, if $\alpha_{2}=\alpha_{3}=\cdots=\alpha_{m}$ then $\alpha$-weighted-bribery is in $\mathrm{P}$; otherwise it is $\mathrm{NP}$-complete.

If $\alpha_{2}=\alpha_{3}=\cdots=\alpha_{m}$ then either the $\alpha$-weighted-bribery is trivially in $\mathrm{P}$ (if $\alpha_{1}=$ $\cdots=\alpha_{m}$ ) or can be solved using the algorithm for plurality-weighted-bribery. The core of the proof is to show NP-hardness. It would be nice to do so by reducing from the corresponding manipulation problems (which share the characterization's boundary line regarding the " $\alpha$ "s). This seems not to work, but in Lemma 4.11 we construct such a reduction that has the right properties whenever its inputs satisfy an additional condition, namely, that the weight of the lightest manipulating voter is at least double that of the heaviest nonmanipulator. This would suffice if the thus-restricted manipulation problem were NP-hard. Lemma 4.12 shows that the thus-restricted manipulation problem is NPhard. It does so by examining the manipulation-dichotomy proof of Hemaspaandra and Hemaspaandra (2007) and noting that if we apply that paper's reduction to Partition' (see Section 2.3) rather than to Partition then we can guarantee the restriction mentioned above.

Definition 4.10 By $\alpha$-weighted-manipulation' we mean the manipulation problem $\alpha$ weighted-manipulation with the restriction that each manipulative voter has weight at least twice as high as the weight of the heaviest of the nonmanipulative voters. Each instance where the restriction is violated is considered not to be an element of $\alpha$-weightedmanipulation'.

Lemma 4.11 Let $\alpha=\left(\alpha_{1}, \ldots, \alpha_{m}\right)$ be a scoring protocol. $\alpha$-weighted-manipulation' $\leq_{m}^{p}$ $\alpha$-weighted-bribery.

Proof. Without loss of generality we can assume that $\alpha_{m}=0$. If $\alpha_{m} \neq 0$ then we can consider the scoring protocol $\alpha^{\prime}=\left(\alpha_{1}-\alpha_{m}, \alpha_{2}-\alpha_{m}, \ldots, \alpha_{m}-\alpha_{m}\right)$ instead. Given an instance $M=(C, V, S, p)$ of the manipulation problem, we construct $B=\left(C, V^{\prime}, p,\|S\|\right)$, a 
bribery instance, such that there is a successful manipulation within $M$ if and only if there is a successful bribery within $B$. We assume that $M$ fulfills $\alpha$-weighted-manipulation's requirements regarding relative weights of voters in $V$ and $S$. If not, we output some fixed $B$ that has no successful briberies.

The reduction works by constructing $V^{\prime}=V \cup S^{\prime}$, where $S^{\prime}$ is the set of voters from $S$ with a fixed arbitrary preference list that has $p$ as the least preferred candidate. Clearly, if a manipulation is possible within $M$ then some bribery works for $B$. We show that the other direction also holds by arguing that if a successful bribery within $B$ exists, then there is a successful bribery that affects only voters in $S^{\prime}$. This implies that $S$ can be viewed as being the manipulative group.

Let us assume that there is some way of bribing at most $\|S\|$ voters in $V^{\prime}$ so that $p$ becomes a winner. If all the bribed voters are in $S^{\prime}$ then the theorem is proven. Otherwise, select some bribed voter $v \in V^{\prime}-S^{\prime}$. By bribing $v, p$ gains at most $\left(\alpha_{1}+\alpha_{1}\right) \cdot \omega(v)$ points over each candidate $c \neq p$. (The first $\alpha_{1}$ is because $p$ can get at most $\alpha_{1}$ additional points by this bribery, and the second $\alpha_{1}$ is because $c$ can lose at most $\alpha_{1}$ votes.) However, if instead of bribing $v$ we would bribe some voter $v^{\prime}$ in $S^{\prime}, p$ would gain at least $\alpha_{1} \omega\left(v^{\prime}\right)$ points over each $c$. (We would bribe $v^{\prime}$ to put $p$ as his or her most preferred candidate and shift all other candidates back.) Since it holds that $\omega\left(v^{\prime}\right) \geq 2 \omega(v)$, we might just as well bribe $v^{\prime}$ instead of $v$, and $p$ would still be a winner. Thus, if $p$ can be made a winner, then $p$ can be made a winner by bribing only voters in $S^{\prime}$.

This reduction can easily be computed in polynomial time.

It remains to show that the restricted version of manipulation is NP-complete for all scoring protocols for which the nonrestricted version is.

Lemma 4.12 If $\alpha=\left(\alpha_{1}, \ldots, \alpha_{m}\right)$ is a scoring protocol such that it is not the case that $\alpha_{2}=\alpha_{3}=\cdots=\alpha_{m}$, then $\alpha$-weighted-manipulation' ${ }^{\prime}$ is NP-complete.

Proof. Let $\alpha=\left(\alpha_{1}, \ldots, \alpha_{m}\right)$ be a scoring protocol such that $\alpha_{2} \neq \alpha_{m}$. We will use Hemaspaandra and Hemaspaandra's (2007) proof of their result that is here called Theorem 4.7 to show the NP-completeness of $\alpha$-weighted-manipulation'. Clearly, $\alpha$-weighted-manipulation' is in NP so we only need to prove its NP-hardness.

Hemaspaandra and Hemaspaandra's (2007) proof of Theorem 4.7 reduces Partition (restricted to positive integers) to $\alpha$-weighted-manipulation. A close inspection of that proof ${ }^{10}$ shows that there exist constants $c$ and $d \geq 2$ that depend only on $\alpha$ such that for every sequence of positive integers $s_{1}, \ldots, s_{n}$ such that $\sum_{i=1}^{n} s_{i}=2 S$, the HemaspaandraHemaspaandra reduction outputs a manipulation problem that has the following properties.

1. Each nonmanipulative voter has weight at most $c S$, and

2. the weights of the manipulative voters are $d s_{1}, d s_{2}, \ldots, d s_{n}$.

We will use these facts to provide a reduction from Partition' to $\alpha$-weighted-manipulation'.

Our reduction works as follows. Let $s_{1}, \ldots, s_{n}$ be the input sequence of nonnegative integers, $\sum_{i=1}^{n} s_{i}=2 S$, such that for each $i, 1 \leq i \leq n$, it holds that $s_{i} \geq \frac{2}{2+n} S$. (As per footnote 3 , if these conditions do not hold then we return a fixed string not in $\alpha$-weighted-

10. We do not repeat that proof here. Interested readers are referred to the paper of Hemaspaandra and Hemaspaandra (2007). 
manipulation'.) Without loss of generality, we assume that $S>0$, and thus $s_{1}, \ldots, s_{n}$ are positive integers. Let $f$ be the reduction given by the proof of Theorem 4.7 in the paper of Hemaspaandra and Hemaspaandra (2007). We compute $f\left(\left(s_{1}, \ldots, s_{n}\right)\right)=(C, V, T, p)$. (Reduction $f$ works for general Partition and so, since we already checked the special properties required by Partition', it has to work correctly for our input.) That is, $s_{1}, \ldots, s_{n}$ can be partitioned if and only if there is a successful manipulation of $(C, V, T, p)$. Unfortunately, we cannot just output $(C, V, T, p)$ as it does not necessarily fulfill the condition on voters' weights. Recall that we have to ensure that each manipulative voter has weight at least twice as high as the weight of the heaviest of the nonmanipulative voters. Let $s_{\min }=\min \left\{s_{j} \mid 1 \leq j \leq n\right\}$. In $(C, V, T, p)$, the least weight of a voter in $T$ is exactly $d s_{\min }$, and the highest weight of a voter in $V$ is at most $c S$. However, we can split each voter $v$ in $V$. The weights of the voters who do not participate in the manipulation are irrelevant as long as the total weight of voters with each given preference order does not change. Thus, we can replace a voter with high weight by several other voters with the same preference order but with lower weights. In our case, we need to make sure that each nonmanipulative voter has at most weight $\frac{1}{2} d s_{\min }$. Since the heaviest of the nonmanipulative voters has weight at most $c S$, we need to replace each voter $v \in V$ by at most

$$
\left\lceil\frac{c S}{\left\lfloor\frac{1}{2} d s_{\min }\right\rfloor}\right\rceil
$$

voters, each of weight at most $\frac{1}{2} d s_{\min }$. Since $d \geq 2, S>0, s_{\min }$ is a positive integer, and $\frac{2}{2+n} S \leq s_{\min }$, we can bound (4) from above by

$$
\left\lceil\frac{c S}{\left\lfloor\frac{1}{2} d s_{\min }\right\rfloor}\right\rceil \leq\left\lceil\frac{c S}{s_{\min }}\right\rceil \leq\left\lceil\frac{c S}{\frac{2 S}{2+n}}\right\rceil=\left\lceil\frac{c(n+2)}{2}\right\rceil,
$$

which is clearly polynomially bounded in $n$. Thus, the splitting of voters can easily be performed in polynomial time, and since it does not change the result of manipulation, the theorem is proven.

The proof of Theorem 4.9 simply combines Lemmas 2.3, 4.11, and 4.12.

Theorem 4.9 shows that bribery within weighted scoring protocols is, in most cases, difficult. Though weighted bribery in light of Theorem 4.9 is easy for trivial elections $\left(\alpha_{1}=\right.$ $\alpha_{m}$ ), plurality, and even plurality's equivalent clones (all scoring systems with $\alpha_{1}>\alpha_{2}=$ $\cdots=\alpha_{m}$ ), if the voters are not only weighted but also have prices then (by Theorem 3.2) bribery also becomes difficult in the case of plurality and plurality's equivalent clones. It is interesting to ask whether having voters who have prices but are not weighted also yields a dichotomy result. As Theorem 4.13 shows, the behavior of scoring protocols with respect to priced voters is very different than with respect to weighted ones.

Theorem 4.13 Let $\alpha=\left(\alpha_{1}, \ldots, \alpha_{m}\right)$ be a scoring protocol. $\alpha$-\$bribery is in $\mathrm{P}$.

Proof. We will give a polynomial-time algorithm for $\alpha$-\$bribery. Let $E=(C, V, p, k)$ be an instance of the problem. First, observe that by considering scoring protocol $\alpha=$ $\left(\alpha_{1}, \ldots, \alpha_{m}\right)$ we, by definition, limit ourselves to a scenario with $m$ candidates, where $m$ is a fixed constant. This implies that there are only a constant number of different preference 
orders, $o_{1}, \ldots, o_{m}$, that the voters might have. We partition $V$ into sets $V_{1}, V_{2}, \ldots, V_{m}$ ! such that each $V_{i}$ contains exactly the voters with preference order $o_{i}$. Some $V_{i}$ 's might be empty and each $V_{i}$ has at most $n$ elements, where $n=\|V\|$.

A bribery within $E$ can be described by giving two sequences of integers, $b_{1}, \ldots, b_{m}$ ! and $d_{1}, \ldots, d_{m !}$, such that $0 \leq b_{i} \leq\left\|V_{i}\right\|$ and $0 \leq d_{i} \leq n$, for $1 \leq i \leq m$ !, and

$$
\sum_{i=1}^{m !} b_{i}=\sum_{i=1}^{m !} d_{i} .
$$

Each $b_{i}$ says how many voters from $V_{i}$ we are bribing. It is sufficient to just give the numbers $b_{i}$ since we want to bribe only the cheapest members of each $V_{i}$. After we bribe these $b=\sum_{i=1}^{m !} b_{i}$ voters, we need to decide what preferences to assign to them. This is described by the sequence $d_{1}, \ldots, d_{m !}$ : Each $d_{i}$ says how many of the $b$ voters will be assigned to have preferences $o_{i}$. Since the voters are indistinguishable, specifying these numbers is enough.

It remains to observe that there are at most $n^{m !}$ sequences $b_{1}, \ldots, b_{m}$ ! and there are at most $n^{m !}$ sequences $d_{1}, \ldots, d_{m}$ ! for each $b$. Thus, there are at most $n^{2(m !)}$ sequences to try out. For each pair of sequences it is easy to check whether after performing the described bribery $p$ becomes a winner and whether the budget is not exceeded. Thus, $\alpha$-\$bribery is in $\mathrm{P}$.

The algorithm given in the proof of Theorem 4.13, with almost no changes, can be used to prove the following corollary.

Corollary 4.14 Let $\mathcal{E}$ be an election system such that (a) for each fixed number of candidates its outcome is computable in polynomial time and (b) its outcome does not depend on the order of the votes. For each fixed number of candidates $\mathcal{E}$-\$bribery is in $\mathrm{P}$.

Theorem 4.13 stands in a sharp contrast to Theorem 4.9. It is natural to ask why prices and weights exhibit such differing behavior. One answer is that in the weighted case the voters retain their individuality - their weights - throughout the whole process of bribery. On the other hand, in the priced case the voters are disassociated from their prices as soon as we decide to bribe them. If we decide to bribe a particular priced voter then we simply need to add his or her price to our total budget, but from then on the voter is indistinguishable from all the other bribed ones. Precisely this observation facilitated the proof of Theorem 4.13 .

The algorithm given in the proof of Theorem 4.13 has a rather disappointing running time. While $n^{O(m !)}$ is a polynomial in our setting, one would certainly prefer to have an algorithm whose time complexity did not depend on $m$ in this way. In particular, it would be nice to have an algorithm with running time polynomial in $n+m$. However, if such an algorithm exists then $\mathrm{P}=\mathrm{NP}$. This follows from the proof of the fact that approval-bribery is NP-complete. In that proof we showed how to reduce X3C to approval-bribery in such a way that each voter approves of at most 3 candidates. If there were a polynomial $p$ and an algorithm that ran in time $p(\|C\|+\|V\|)$ for every scoring protocol $\alpha$, then we could solve X3C by reducing it to approval-bribery and then embedding that approval-bribery problem in an $\alpha$-bribery problem for some $\alpha=(1,1,1,0, \ldots, 0)$, possibly adding some dummy candidates. This embedding is straightforward so we do not describe it in detail. 
Let $\alpha=\left(\alpha_{1}, \ldots, \alpha_{m}\right)$ be a scoring protocol such that it is not the case that $\alpha_{2}=$ $\cdots=\alpha_{m}$. By Theorem 4.9 we know that $\alpha$-weighted-bribery is NP-complete. We also know, by Theorem 4.13 , that $\alpha$-\$bribery is in P. It clearly holds that $\alpha$-weighted-\$bribery is NP-complete, but it is interesting to ask whether the NP-completeness of $\alpha$-weightedbribery and $\alpha$-weighted-\$bribery holds because of the possibly exponentially large values of the weights, or do these problems remain NP-complete even if the weights are encoded in unary? It turns out, by the following theorem, that high weight values are necessary for NP-completeness.

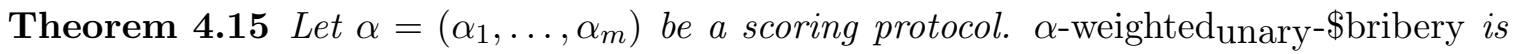
in $\mathrm{P}$.

Proof. Let $\alpha=\left(\alpha_{1}, \ldots, \alpha_{m}\right)$ be a scoring protocol. The proof of this theorem cashes in on the same observation as that made in the proof of Theorem 4.13: There are only finitely many different preference orders, and there are only polynomially many substantially different ways of bribing.

Let $E=(C, V, p, k)$ be a bribery problem and let $o_{1}, \ldots, o_{m}$ ! be all the different possible preference orders over $C$. We partition $V$ into $m$ ! disjoint sets $V_{1}, \ldots, V_{m}$ ! such that each $V_{i}$ contains exactly the voters with preference order $o_{i}$. A bribery within $E$ can be described by a sequence of $m$ ! vectors $b_{i}=\left(b_{i, 1}, b_{i, 2}, \ldots, b_{i, m !}\right), 1 \leq i \leq m !$, such that for each $i, j$, $1 \leq i, j \leq m !, b_{i, j}$ is a nonnegative integer and for each $i, 1 \leq i \leq m$ !, we have

$$
\sum_{j=1}^{m !} b_{i, j}=\omega\left(V_{i}\right) .
$$

The interpretation of a vector $b_{i}$ is that voters in $V_{i}$ can be partitioned into $m$ ! sets $V_{i, 1}, \ldots, V_{i, m}$ ! such that $\omega\left(V_{i, j}\right)=b_{i, j}$, with the intention of bribing voters in $V_{i, j}$ to change their preference lists to $o_{j}$ (recall that $V_{i}$ is a multiset, and so of course this is a multiset partition and the $V_{i, j}$ 's will be multisets). When $i \neq j$ this bribery has some price, and when $i=j$ it is for free as nothing really needs to be done. Note that not all vectors are realizable; not every splitting of vote weight $\omega\left(V_{i}\right)$ can be achieved. The rest of this proof is devoted to developing a method for evaluating whether a given split is possible and what its minimal cost is. There are only $\left(\omega(V)^{m !}\right)^{m !}$ ways of selecting vectors $b_{1}, \ldots, b_{m}$ ! so if we can test whether a given vector is realizable (and compute the minimal price for its realization), then we can simply try all sequences of vectors and test whether any of them both makes $p$ a winner (the winner, in the unique case) and has its total cost fall within the budget.

We will now describe an algorithm that checks if a particular vector $w=\left(w_{1}, \ldots, w_{m !}\right)$, where $w_{i} \in\{0, \ldots, \omega(V)\}$ for each $i$ in $\{1, \ldots, m !\}$, is realizable and computes the minimal price for $w$ 's realization. By $V_{i}^{\prime}\left(w_{1}, \ldots, w_{m}\right)$ we mean the following set of $m$ !-element sequences of subsets of $V_{i}$ :

$$
V_{i}^{\prime}(w)=\left\{\left(V_{i, 1}, \ldots, V_{i, m !}\right) \mid\left(V_{i}=\bigcup_{j=1}^{m !} V_{i, j}\right) \wedge(\forall 1 \leq j \leq m !)\left[\omega\left(V_{i, j}\right)=w_{j}\right]\right\} .
$$

For each $w$ we define

$$
g_{i}(w)= \begin{cases}\min \left\{\rho \mid\left(\exists\left(V_{i, 1}, \ldots, V_{i, m !}\right) \in V_{i}^{\prime}(w)\right)\left[\rho=\sum_{j \neq i} \pi\left(V_{i, j}\right)\right]\right\} & \text { if } V_{i}^{\prime}(w) \neq \emptyset \\ \infty & \text { otherwise }\end{cases}
$$


That is, $g_{i}(w)$ gives the lowest price for bribing the voters in $V_{i}$ according to weight vector $\left(w_{1}, \ldots, w_{m}\right)$. We can compute $g_{i}(w)$ in polynomial time using dynamic programming techniques. Let us rename the candidates so that $V_{i}=\left\{v_{1}, \ldots, v_{t}\right\}$ and let $g_{i, \ell}(w)$ be the same as $g_{i}(w)$ except restricted to voters $v_{\ell}, \ldots, v_{t}$. Thus, $g_{i, 1}$ is exactly $g_{i}$. Naturally, the following boundary condition holds for $g_{i, t+1}$.

$$
g_{i, t+1}\left(w_{1}, \ldots, w_{m !}\right)= \begin{cases}0 & \text { if } w_{1}=w_{2}=\cdots=w_{m !}=0 \\ \infty & \text { otherwise }\end{cases}
$$

We can compute values of $g_{i, \ell}\left(w_{1}, \ldots, w_{m !}\right)$ using dynamic programming and the observation that $g_{i, \ell}\left(w_{1}, \ldots, w_{m !}\right)$ is equal to the minimum of the following:

$$
\begin{aligned}
& g_{i, \ell+1}\left(w_{1}-\omega\left(v_{\ell}\right), w_{2}, \ldots, w_{m !}\right)+\pi\left(v_{\ell}\right), \\
& g_{i, \ell+1}\left(w_{1}, w_{2}-\omega\left(v_{\ell}\right), w_{3}, \ldots, w_{m !}\right)+\pi\left(v_{\ell}\right), \\
& \ldots \\
& g_{i, \ell+1}\left(w_{1}, \ldots, w_{m !-1}, w_{m !}-\omega\left(v_{\ell}\right)\right)+\pi\left(v_{\ell}\right), \text { and } \\
& g_{i, \ell+1}\left(w_{1}, \ldots, w_{i-1}, w_{i}-\omega\left(v_{\ell}\right), w_{i+1}, \ldots, w_{m !}\right) .
\end{aligned}
$$

Note that the last of the values handles the fact that if we bribe $v_{\ell}$ to report preference order $o_{i}$ then we actually do not need to pay him or her; $v_{\ell}$ already has preference order $o_{i}$. Otherwise, we need to decide which of the $m !-1$ other preference orders we ask $v_{\ell}$ to report, and we need to pay for this change. Clearly, using this rule and the above boundary condition we can compute $g_{i, 1}(w)$, and thus $g_{i}(w)$, in time polynomial in $\omega(V)$. Since $\omega(V)$ is polynomial in the size of the input, this completes the proof.

Corollary 4.16 Let $\mathcal{E}$ be an election system such that (a) for each fixed number of candidates its outcome is computable in polynomial time and (b) its outcome does not depend on the order of the votes. For each fixed number of candidates $\mathcal{E}$-weighted unary-\$bribery is in $\mathrm{P}$.

Note that, by Theorem 4.6, it holds that for each scoring protocol $\alpha$,

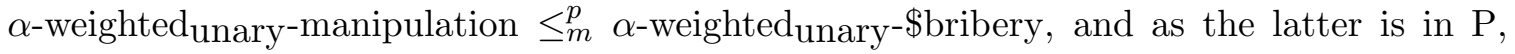
we have the following corollary.

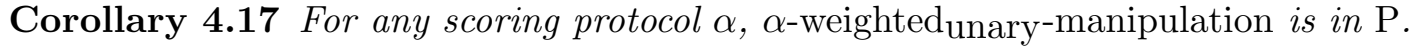

Certain scoring protocols have natural generalizations to an arbitrary number of candidates, e.g., the plurality rule, the Borda count, and the veto rule. Our results above do not imply easiness of bribery for such election systems, as we need a single $\mathrm{P}$ algorithm to work in all cases. For example, for the case of Borda count, recently Brelsford et al. (2008) have shown that even Borda-bribery is NP-complete. In other cases, easiness results can be easily obtained "by hand." For example, Theorem 4.9 immediately implies that vetoweighted-bribery is NP-complete even for 3 candidates, yet the following result shows that the difficulty of bribery for veto voting comes purely from the weighted votes.

Theorem 4.18 veto-bribery is in $\mathrm{P}$. 
Proof. The proof of this theorem is essentially the same as that of Theorem 3.1. We can view veto elections as elections in which every voter vetos one candidate, and a candidate with the least number of vetoes wins. (In the unique case, a candidate is the winner if and only if no other candidate has as few vetoes as he or she has.)

Thus, given an instance $E=(C, V, p, k)$, we keep on bribing voters that veto $p$ and ask them to veto a candidate that, at that time, has the least number of vetoes. If after at most $k$ bribes $p$ is a winner then we accept; otherwise we reject. A simple inductive argument shows this is a correct strategy, and the algorithm clearly runs in polynomial time.

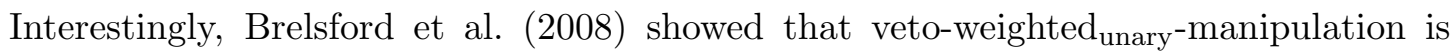
NP-complete. This immediately gives, by Theorem 4.6, that veto-weighted unary $_{\text {- }}$ bribery is NP-complete. Using techniques similar to those we used in the proof of Theorem 4.9 (but much simpler) we can modify their reduction to show that even veto-weighted unary $^{-}$ bribery is NP-complete. On the other hand, the main result of the paper Faliszewski (2008) implies that veto-\$bribery is in $\mathrm{P}$. As to whether Theorem 4.18 follows immediately from Theorem 4.13, it does not. Why? Recall that 4.13 covers veto for each fixed number of candidates, but Theorem 4.18 is covering the protocol that handles veto for all numbers of candidates - what people commonly think of when they think of veto as a voting system.

We in spirit obtained Theorem 4.9 by reducing (with much work and adjustment) from manipulation to bribery, for scoring protocols. Will that work in all other settings? The answer is no; we have designed an artificial voting system where checking manipulability even by just one voter is NP-complete, but checking bribability is easy.

Theorem 4.19 There exists a voting system $\mathcal{E}$ for which manipulation is NP-complete, but bribery is in $\mathrm{P}$.

Proof. Let $A$ be an NP-complete set and let $B \in \mathrm{P}$ be such that

1. $A=\left\{x \in \Sigma^{*} \mid\left(\exists y \in \Sigma^{*}\right)[\langle x, y\rangle \in B]\right\}$, and

2. $\left(\forall x, y \in \Sigma^{*}\right)[\langle x, y\rangle \in B \Rightarrow|x|=|y|]$.

Such sets can easily be constructed from any NP-complete set by padding. The idea of the proof is to embed a verifier for $A$ within the election rule $\mathcal{E}$. We do this in a way that forces manipulation to solve arbitrary $A$ instances, while allowing bribery to still be easy.

First, we observe that preference lists can be used to encode arbitrary binary strings. We will use the following encoding. For $C$ a set of candidates, let $c_{1}, c_{2}, \ldots, c_{m}$ be those candidates in lexicographical order. We will view the preference list

$$
c_{i_{1}}>c_{i_{2}}>c_{i_{3}}>\cdots>c_{i_{m}}
$$

as an encoding of the binary string $b_{1} b_{2} \cdots b_{\lfloor m / 2\rfloor}$, where

$$
b_{j}= \begin{cases}0 & \text { if } i_{2 j-1}>i_{2 j} \\ 1 & \text { otherwise }\end{cases}
$$

This encoding is of course not the most efficient one, and a given binary string may have many preference lists that encode it. However, this encoding is very easy and has the properties that we need in our construction. 
In our reduction, binary strings starting with 1 will encode instances, and binary strings starting with 0 will encode witnesses. Given this setup, we can describe our election system $\mathcal{E}$. Let $(C, V)$ be an election. For each $c \in C, c$ is a winner of the election if and only if $\|V\|=3$ and

Rule 1: all preference lists encode strings starting with 1 or all preference lists encode strings starting with 0 , or

Rule 2: exactly one preference list encodes a string that starts with 1 , say $1 x$, and at least one other preference list encodes a string $0 y$ such that $\langle x, y\rangle \in B$.

Thus, either all candidates are winners or none of them are winners. Note that testing whether a candidate $c$ is a winner of an $\mathcal{E}$ election can easily be done in polynomial time. The following polynomial-time algorithm shows how to perform an optimal bribery. This implies that $\mathcal{E}$-bribery $\in \mathrm{P}$.

1. If $c$ is a winner, we do nothing.

2. Otherwise, if $\|V\| \neq 3$, then bribery is impossible.

3. Otherwise, if there is exactly one voter whose preference list encodes a string that starts with 1, then we bribe that voter to encode a string that starts with 0 . By Rule $1, c$ is a winner of the election.

4. Otherwise, there is exactly one voter whose preference list encodes a string that starts with 0 and we bribe that voter so that his or her preference list encodes a string that starts with 1. By Rule 1, $c$ is a winner of the election.

On the other hand, the ability to solve the manipulation problem for $\mathcal{E}$ implies the ability to solve $A$. We construct a reduction from $A$ to $\mathcal{E}$-manipulation. Given a string $x \in \Sigma^{*}$, we first check whether $\left\langle x, 0^{|x|}\right\rangle \in B$. If so, then clearly $x \in A$ and we output some fixed member of $\mathcal{E}$-manipulation. Otherwise, we output a manipulation problem with candidates $\{1,2, \ldots, 2(|x|+1)\}$ and three voters, $v_{0}, v_{1}$, and $v_{2}$, such that $v_{0}$ 's preference list encodes $1 x, v_{1}$ 's preference list encodes $00^{|x|}$, and $v_{2}$ is the only manipulative voter. We claim that candidate 1 can be made a winner if and only if $x \in A$.

Since $\left\langle x, 0^{|x|}\right\rangle \notin B$, the only way in which $v_{2}$ can make 1 a winner is when $v_{2}$ encodes a string $0 y$ such that $\langle x, y\rangle \in B$ in which case $x \in A$. For the converse, if $x \in A$, there exists a string $y \in \Sigma^{|x|}$ such that $\langle x, y\rangle \in B$. We can encode string $0 y$ as a preference list over $\{1,2, \ldots, 2(|x|+1)\}$, and let this be the preference list for $v_{2}$. This ensures that 1 is a winner of the election.

Since this reduction can be computed in polynomial time, and the $\mathcal{E}$-manipulation's membership in NP is clear, we have that $\mathcal{E}$-manipulation is NP-complete.

The same result holds for the case of unique winners. In this case we modify $\mathcal{E}$ such that only the lexicographically smallest candidate can win the election and the reduction will define the distinguished candidate as the lexicographically smallest candidate.

The above election system is not natural, but it does show that unless we restrict our election rules somehow or prove $\mathrm{P}=\mathrm{NP}$, obtaining a general reduction from manipulation to bribery seems precluded. 


\section{Succinct Elections}

So far we have discussed only nonsuccinct elections - ones where voters with the same preference lists (and weights, if voters are weighted) are given by listing each of them one at a time (as if given a stack of ballots). It is also very natural to consider the case where each preference list has its frequency conveyed via a count (in binary), and we will refer to this as "succinct" input. The succinct representation is particularly relevant in case when the number of candidates is bounded by a constant. When there are many candidates, it is natural to expect that a lot of voters will have preferences that vary in some insignificant ways. On the other hand, if there are very few candidates then, naturally, there will be large numbers of voters with the same preferences, and using succinct representation will save a lot of space.

In this section we provide $\mathrm{P}$ membership results (and due to our proofs, these in fact each are even FPT membership results ${ }^{11}$ ) regarding bribery in succinctly represented elections with a fixed number of candidates. Our main tool here is Lenstra's (1983) extremely powerful result that the integer programming feasibility problem is in $\mathrm{P}$ if the number of variables is fixed.

Theorem 5.1 (Lenstra, 1983) Let $k$ be some fixed nonnegative integer. There is a polynomial-time algorithm that given an $m \times k$ integer matrix $A$ and a vector $b \in \mathbb{Z}^{m}$ determines whether

$$
\left\{x \in \mathbb{Z}^{k} \mid A x \leq b\right\} \neq \emptyset
$$

holds. That is, integer linear programming is in $\mathrm{P}$ for a fixed number of variables.

We mention that Lenstra's polynomial-time algorithm is not at all attractive practically speaking. In particular, although the algorithm uses just a linear number of arithmetic operations on linear-sized integers - and thus it has a theoretically attractive, low-degree polynomial run-time - the multiplicative constant is very large. To us here this is not a critical issue since we are mostly interested in polynomial-time computability results and general tools for obtaining them, rather than in actual optimized or optimal algorithms.

Although Lenstra's result applies to the integer linear programming problem when the number of variables is fixed and achieves $\mathrm{P}$-time in that case, in this section we in fact

11. Regarding the natural issue of which $\mathrm{P}$ results can be strengthened to being FPT results, we mention in passing that every $\mathrm{P}$ membership result of this section is clearly (although implicitly), via its proof, even an FPT membership result. (A problem with some parameter $j$ is in FPT, a class capturing the notion of being fixed-parameter tractable, if there exists an algorithm whose running time on instances of size $n$ is bounded by $f(j) n^{O(1)}$, where $f$ is some function depending only on $j$; see Niedermeier, 2006, for detailed coverage of parameterized complexity.) Essentially, this is because Lenstra's method is well known to use a linear number of arithmetic operations on linear-sized variables (Lenstra, 1983, see also Downey, 2003; Niedermeier, 2002). Although the fact that some voting problems are in FPT is implicit in the seminal work of Bartholdi et al., 1989b (e.g., see Christian, Fellows, Rosamond, \& Slinko, 2007; Betzler, Guo, \& Niedermeier, 2008b), we mention that the work of Christian et al. (2007, Section 4), which itself has a bribery-like flavor to its results, explicitly addresses the issue of FPT, in particular mentioning that it is well known that the (nonsuccinct) winner and score problems for Kemeny and Dodgson are in FPT, and we are indebted to an earlier version of their paper as it motivated us to here mention that this section's P results (even those about succinct elections) are FPT results. Among the other papers addressing FPT results for election problems (although not regarding bribery problems), we mention as examples the work of Betzler, Fellows, Guo, Niedermeier, and Rosamond (2008a) on Kemeny voting and of Faliszewski et al. (2008) on Llull and Copeland voting. 
typically only need the special case of his result in which the number of variables and the number of constraints are both fixed (and so the only parameter that is changing is the constants within the constraints).

$\mathrm{P}$ membership results regarding succinctly represented elections naturally imply analogous results for the nonsuccinct representation. To express the fact that succinctness of representation is optional in such cases, we put the phrase succinct in curly braces in the names of the problems. For example, if we say that plurality-\{succinct $\}$-bribery is in $\mathrm{P}$, we mean that both plurality-bribery and plurality-succinct-bribery are in P. (By the way, Theorem 3.1, by a similar but more careful algorithm than the one in its proof also holds for the succinct case.)

Before we proceed with the results, let us introduce some notation. Throughout this section we assume that all bribery problems that we are dealing with have exactly $m$ candidates, where $m$ is some arbitrary fixed constant. Thus, if $E=(C, V, p, k)$ is a bribery problem then we may assume that $C=\{1, \ldots, m\}$, and that $o_{1}, o_{2}, \ldots, o_{m}$ ! are all the possible preference orders over $C$. Given a set of voters $V$, by $V_{i}, 1 \leq i \leq m$ !, we mean the set of voters $v \in V$ that have preference order $o_{i}$. For a given $i$, we define $w h(c, i)$ to be the index of candidate $c$ within preferences $o_{i}$ (informally, where is $c$ in $o_{i}$ ). This notation is assumed in each of the proofs of this section.

Using the integer programming approach we obtain polynomial-time algorithms for bribery under scoring protocols in both the succinct and the nonsuccinct cases. The same approach yields a similar result for manipulation. (The nonsuccinct case for manipulation was already obtained in the work of Conitzer et al., 2007.)

Theorem 5.2 For every scoring protocol $\alpha=\left(\alpha_{1}, \ldots, \alpha_{m}\right)$, both $\alpha$-\{succinct $\}$-bribery and $\alpha$-\{succinct $\}$-manipulation are in $\mathrm{P}$.

Proof. Let $\alpha=\left(\alpha_{1}, \ldots, \alpha_{m}\right)$ be a scoring protocol and let $E=(C, V, p, k)$ be the bribery problem we want to solve, where $C=\{1, \ldots, m\}$. A bribery can be described by providing numbers $s_{i, j}, 1 \leq i, j \leq m$ !, saying how many people should switch from preference order $o_{i}$ to preference order $o_{j}$. (The values $s_{i, i}$ simply say how many voters with preference order $o_{i}$ should not switch to anything else. And we do allow superfluous exchanging, e.g., it is legal, even when $i \neq j$, to have both $s_{i, j}$ and $s_{j, i}$ be strictly greater than zero. However, note that in this proof, for example, if there is a solution where that happens then there will be another solution in which it holds that, for each $i \neq j$, at least one of $s_{i, j}$ and $s_{j, i}$ is zero.) We may express by an integer program the fact that the $s_{i, j}$ 's describe a successful bribery, and we do so as follows. Here, our variables, the $s_{i, j}$ 's, are required to be integers. We have $(m !)^{2}$ such variables. Our constants (they are constants-i.e., coefficients - from the integer linear programming perspective, not all are constants from the complexity perspective, as in effect $k$, and $\left\|V_{1}\right\|, \ldots,\left\|V_{m !}\right\|$ are inputs to the problem) are $k, \alpha_{1}, \alpha_{2}, \ldots, \alpha_{m},\left\|V_{1}\right\|,\left\|V_{2}\right\|, \ldots,\left\|V_{m !}\right\|$.

1. The number of bribed voters has to be nonnegative. For all $i, j, 1 \leq i, j \leq m$ !, we have

$$
s_{i, j} \geq 0
$$

2. We cannot bribe more voters with a given preference than there are. For each $i$, $1 \leq i \leq m$ !, we have the constraint (keeping in mind that the $s_{i, i}$ 's pick up any 
leftover, thus we state this as an equality)

$$
\sum_{j=1}^{m !} s_{i, j}=\left\|V_{i}\right\|
$$

3. Altogether, we can only bribe at most $k$ people.

$$
\sum_{i=1}^{m !} \sum_{j=1}^{m !} s_{i, j}-\sum_{\ell=1}^{m !} s_{\ell, \ell} \leq k .
$$

4. The score of $p$ is at least as high as anybody else's. For each $h, 1 \leq h \leq m$, we have a constraint that says that candidate $h$ does not defeat $p$ :

$$
\sum_{j=1}^{m !} \alpha_{w h(p, j)}\left(\sum_{i=1}^{m !} s_{i, j}\right) \geq \sum_{j=1}^{m !} \alpha_{w h(h, j)}\left(\sum_{i=1}^{m !} s_{i, j}\right) .
$$

We have a constant number of variables, $(m !)^{2}$, and a constant number of constraints. (Of course, on the other hand the size of some of our integer linear program's "constants" in particular $k,\left\|V_{1}\right\|, \ldots,\left\|V_{m !}\right\|$-may increase with the number of voters.) Thus using Lenstra's algorithm we can in polynomial time test whether the above set of constraints can be satisfied by some legal $s_{i, j}$ 's. It is clear that these constraints can be satisfied if and only if there is a bribery of at most $k$ voters that leads to making $p$ a winner. Also, note that to make this program test whether $p$ can become a unique winner we simply make the inequalities in the final set of constraints strict.

The case of manipulation can be proved very similarly, only that we would have variables $s_{i}$ that would say how many of the manipulators decide to report preference order $o_{i}$. We omit the detailed description as it is clear given the above.

The power of the integer programming approach is not limited to the case of scoring protocols. In fact, the seminal paper of Bartholdi et al. (1989b) shows that applying this method to computing the Dodgson score in nonsuccinct elections with a fixed number of candidates yields a polynomial-time score algorithm (and though the paper of Bartholdi et al., 1989b, did not address the issue of succinct elections, one can see that there too this method works perfectly; that is, it is implicit from the "Lenstra" approach of the paper of Bartholdi et al., 1989b, that Dodgson score for elections with a fixed number of candidates is, even for the succinct case, in FPT, see also footnote 11). ${ }^{12}$ A similar program can be used to compute the scores within Young elections. Let us recall the definition of both Dodgson and Young scores.

Definition 5.3 Given a set of candidates $C$ and a set of voters $V$ specified via their preferences, the Dodgson score of a candidate $c \in C$ is the minimum number of adjacent switches within the preferences of voters that make $c$ a Condorcet winner.

The Young score of a candidate $c \in C$ is the minimum number of voters that need to be removed from the elections to make $c$ a Condorcet winner.

12. We mention in passing that some recent work responds to the theoretical complexity of Dodgson scores from a different direction, namely, by studying the success rate of simple heuristics for the problem (McCabe-Dansted et al., 2008; Homan \& Hemaspaandra, 2009). 
Applying the integer programming attack for the case of bribery within Dodgson-like election systems, i.e., the Dodgson system and the Young system, is more complicated. These systems involve a more intricate interaction between bribing the voters and then changing their preferences. For Dodgson elections, after the bribery, we still need to worry about the adjacent switches within voters' preference lists that make a particular candidate a Condorcet winner. For Young elections, we need to consider the voters being removed from the elections. This interaction seems to be too complicated to be captured by an integer linear program, but building on the flavor of the Bartholdi et al. (1989b) integer programming attack we can achieve the following: Instead of making $p$ a winner, we can attempt to make $p$ have at most a given Dodgson or Young score.

Formally, by DodgsonScore-bribery (and by its succinctly encoded variant, DodgsonScore-succinct-bribery) we mean the problem that takes as input a Dodgsonbribery instance $(C, V, p, k)$ (with voters encoded succinctly in the succinct variant) and a nonnegative integer $t$, and asks if it is possible to ensure - by bribing at most $k$ voters - that $p$ 's Dodgson score is at most $t$. We define YoungScore-bribery and YoungScore-succinctbribery analogously.

Theorem 5.4 For each fixed number of candidates, DodgsonScore-\{succinct\}-bribery is in $\mathrm{P}$ when restricted to that number of candidates.

Proof. Given a nonnegative integer $t$ and a bribery problem instance $E=(C, V, p, k)$ for Dodgson elections, where $C=\{1, \ldots, m\}$, we will give an integer program that tests whether it is possible to bribe at most $k$ voters in such a way that, after the bribery, $p$ has Dodgson score at most $t$. Our program will have only a constant number of variables and a constant number of constraints. Thus by Lenstra's algorithm it can be solved in polynomial time.

The process of bribery has, in the case of Dodgson elections, two phases: a bribery phase in which we decide how to bribe voters, and a swapping phase in which we (in effect) allow at most $t$ adjacent swaps to occur. We will model the first phase with integer variables $b_{i, j}$ and the second phase with integer variables $s_{i, j}$ : For each $i, j, 1 \leq i, j \leq m$ !, the interpretation of $b_{i, j}$ and $s_{i, j}$ is as follows.

$b_{i, j}$ - the number of voters with preference order $o_{i}$ who are bribed to report preference order $o_{j}$.

$s_{i, j}$ - the number of voters who, after the bribery, change their preference order from $o_{i}$ to $o_{j}$.

The values $b_{i, i}$ say how many voters with preferences $o_{i}$ are not bribed. The values $s_{i, i}$ say how many voters with preferences $o_{i}$ do not change their preferences. We have these variables $b_{i, i}$ and $s_{i, i}$ as they make our equations neater.

Recall that the Dodgson score is the number of adjacent switches within preference lists that are needed to make a given candidate a Condorcet winner. However, the variables $s_{i, j}$ talk about much more complicated operations, namely transfers from preference order $o_{i}$ to preference order $o_{j}$. For each $i, j, 1 \leq i, j \leq m$ !, define a constant switches $s_{i, j}$ which gives the minimum number of adjacent switches that lead from preference order $o_{i}$ to preference 
order $o_{j} .{ }^{13}$ For every preference order $o_{i}$ and every two candidates $r$ and $q$ we define who $(r, q, i)$ to be 1 if $r$ strictly defeats $q$ in the preference order $o_{i}$ and to be -1 otherwise. $w h o(r, r, i)=-1$, but we will never invoke this fact. Our integer linear program has the following constraints.

1. The number of bribes and switches has to be nonnegative. For each $i, j, 1 \leq i, j \leq m$ !, we have

$$
\begin{aligned}
& b_{i, j} \geq 0, \text { and } \\
& s_{i, j} \geq 0 .
\end{aligned}
$$

2. We cannot bribe more voters than there are. For each $i, 1 \leq i \leq m$ !, we require

$$
\sum_{j=1}^{m !} b_{i, j}=\left\|V_{i}\right\|
$$

3. Altogether, we can bribe at most $k$ people.

$$
\sum_{i=1}^{m !} \sum_{j=1}^{m !} b_{i, j}-\sum_{\ell=1}^{m !} b_{\ell, \ell} \leq k .
$$

4. The number of voters who switch from preference order $o_{i}$ in the swapping phase needs to be equal to the number of voters who, after the bribery, had preference order $o_{i}$. For each $i, 1 \leq i \leq m$ !,

$$
\sum_{j=1}^{m !} b_{j, i}=\sum_{k=1}^{m !} s_{i, k}
$$

5. After the swapping phase, $p$ is a Condorcet winner. For every $q \in C-\{p\}$,

$$
\sum_{i=1}^{m !} \sum_{j=1}^{m !} w h o(p, q, j) \cdot s_{i, j}>0 .
$$

6. The swapping phase involves at most $t$ adjacent switches within preference lists.

$$
\sum_{i=1}^{m !} \sum_{j=1}^{m !} \text { switches }_{i, j} \cdot s_{i, j} \leq t .
$$

13. The astute reader will note that to seek to meet or beat a given score for $p$ under a given amount of bribery, one would never need to in the Dodgson score calculation invoke any exchanges that do anything except move $p$ ahead some number of slots. This is true, and thus rather than our $(m !)^{2}$ variables $s_{i, j}$, one could define an integer linear program that replaced the $s_{i, j}$ 's with $(m !)(m-1)$ variables that capture just such shifting. We define things in the current more general way since the current approach removes the dependence on a "we can get away with just shifts of this sort" argument of the type just made (which would work fine here but might not hold in sharply different settings), and the current approach also leads to quite simple, uncluttered constraint equations. 
Clearly, this program contains a constant number of variables and a constant number of constraints. Thus in light of Lenstra's algorithm the theorem is proven.

Theorem 5.5 For each fixed number of candidates, YoungScore-\{succinct\}-bribery is in $\mathrm{P}$ when restricted to that number of candidates.

Proof. The proof is very similar to the proof of Theorem 5.4. Let $t$ be a nonnegative integer and let $E=(C, V, p, k)$ be a bribery instance where $C=\{1, \ldots, m\}$. We want to provide an algorithm that tests whether it is possible to ensure that $p$ has Young score at most $t$ by bribing at most $k$ voters. We will do so by providing an integer linear program.

The workings of the integer linear program are be divided into two phases: the bribery phase and the removal phase. The bribery is described by variables $b_{i, j}, 1 \leq i, j \leq m$ !, which say how many voters with preferences $o_{i}$ are bribed to have preferences $o_{j}$. The removal is described by variables $r_{i}, 1 \leq i \leq m$ !, which say how many of the voters who have preferences $o_{i}$ after the bribery are being removed. To enforce the above, we use the following constraints:

1. The number of bribes and removals has to be nonnegative. For each $i, j, 1 \leq i, j \leq m$ !, we have

$$
\begin{aligned}
b_{i, j} & \geq 0, \text { and } \\
r_{i} & \geq 0 .
\end{aligned}
$$

2. We cannot bribe more voters than there are. For each $i, 1 \leq i \leq m$ !, we have

$$
\sum_{j=1}^{m !} b_{i, j}=\left\|V_{i}\right\|
$$

3. Altogether, we can only bribe at most $k$ people.

$$
\sum_{i=1}^{m !} \sum_{j=1}^{m !} b_{i, j}-\sum_{\ell=1}^{m !} b_{\ell, \ell} \leq k .
$$

4. The number of voters with preference order $o_{i}$ who are removed from the election during the removal phase has to be bounded by the number of voters who after the bribery have preference order $o_{i}$. For each $i, 1 \leq i \leq m$ !, we have

$$
r_{i} \leq \sum_{j=1}^{m !} b_{j, i}
$$

5. After the removal phase, $p$ is a Condorcet winner. For every $q \in C-\{p\}$,

$$
\sum_{j=1}^{m !}\left(\left(\sum_{i=1}^{m !} b_{i, j}\right)-r_{j}\right) \cdot w h o(p, q, j)>0 .
$$


6. The removal phase removes at most $t$ voters.

$$
\sum_{i=1}^{m !} r_{i} \leq t .
$$

Clearly, there are a constant number of variables and constraints, so the integer linear program can be solved using Lenstra's algorithm in polynomial time.

The above two theorems say that we can test in polynomial time whether a given bribe suffices to obtain or beat a given Dodgson or Young score. Thus using binary search we can in fact find the optimal bribe for obtaining a particular score.

The issue of actually making a candidate $p$ a winner (a unique winner, if we are studying the unique winner case) of Dodgson elections is, as already indicated, much more difficult and a direct attack using integer linear programming seems to fail. Nonetheless, combining the integer programming method with a brute-force algorithm resolves the issue for the nonsuccinct case.

Theorem 5.6 For each fixed number of candidates, Dodgson-bribery, Dodgson-\$bribery, Young-bribery, and Young-\$bribery are all in $\mathrm{P}$.

Proof. As in Theorem 4.13, there are only polynomially many briberies we need to check. For each of them we test whether our favorite candidate becomes a winner, using Bartholdi et al.'s (1989b) integer linear program for Dodgson score-testing or a similar one for Young score-testing.

The above discussions of bribery with respect to Dodgson elections lead to an observation that a small change in the voting system does allow us to resolve a natural bribery-related winner problem. Note that bribes allow us to completely change a given voter's preference list - and this goes far beyond the switches allowed by Dodgson score-counting. It is interesting to observe that one can define a Dodgson-like voting system based on bribes: Instead of counting how many switches are needed to make a given candidate the Condorcet winner, we count how many bribes (where each bribe is a complete overwrite, at unit cost, of one voter's preference list) suffice to guarantee such an outcome. We call this election system

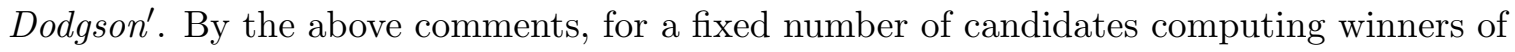
Dodgson' elections can be done in polynomial time.

Theorem 5.7 For each fixed number of candidates, the winner problem for succinct Dodgson' elections is in $\mathrm{P}$.

Proof. This follows immediately from Theorem 5.4. For each candidate $c$ we simply need to binary search for the smallest bribe that makes him or her a Condorcet winner (i.e., gives $c$ Dodgson score zero). The winners are those candidates for whom the least number of bribes is needed.

Clearly, like Dodgson, Dodgson' elects the Condorcet winner whenever one exists. Theorem 5.7 shows that for each fixed number of candidates the winner problem in succinct Dodgson' elections is in $\mathrm{P}$, and just before Definition 5.3 we noted that the same holds for succinct Dodgson elections. As to which is more attractive, that will depend on what one feels the more natural model is for counting distance - counting each adjacent switch or counting each voter who had any change. In settings in which the latter seems more 
attractive, of course Dodgson' seems preferable to Dodgson. Nonetheless, before jumping on any Dodgson' bandwagon, one should probably first carefully study the properties of the new election system. Note that even though computing Dodgson' winners for a fixed number of candidates is a polynomial-time procedure, this does not immediately imply that the bribery problem is easy for Dodgson', and we conjecture that it is not.

In light of the above discussion, it might seem that for Dodgson-like election rules getting polynomial-time bribery results (in the succinct model) is very difficult using integer linear programming. However, this is not always the case. In particular, the following theorem states that bribery in the Kemeny system is easy if we fix the number of candidates. Recall that a candidate $c$ is a winner of the Kemeny elections if there exists a preference order $o_{h}$ that lists $c$ on top and that "agrees" most strongly with the votes. (See Section 2.1.)

Theorem 5.8 For each fixed number of candidates, Kemeny-\{succinct\}-bribery is in $\mathrm{P}$ when restricted to that number of candidates.

Proof. The proof employs integer linear programming, but this time we need more than just one program. Very informally put, this is because integer linear programs seemingly can express only conjunctions, but not disjunctions, and in the case of Kemeny elections we need to express the fact that at least one of the preference orders that lists our favorite candidate on top disagrees with the least number of voters' preferences. $^{14}$

Let $E=(C, V, p, k)$ be a bribery instance for Kemeny elections, where $C=\{1, \ldots, m\}$. For each preference order $o_{h}, 1 \leq h \leq m$ !, such that $p$ is the top candidate in $o_{h}$, we construct a separate integer linear program that has a feasible solution if and only if there is a bribery of at most $k$ candidates after which $o_{h}$ is an ordering that maximizes (compared against all other orders) the number of agreements with voters' reported preferences. By agree $_{i, j}$ we mean the number of agreements between preference orders $o_{i}$ and $o_{j}$ (see Section 2.1).

Let us consider an arbitrary $h$ such that $p$ is the top candidate in preference order $o_{h}$. We describe the bribery using variables $b_{i, j}, 1 \leq i, j \leq m$ !, each saying how many voters with preference order $o_{i}$ are bribed to have preference order $o_{j}$. We employ the following constraints.

1. The number of bribes has to be nonnegative. For each $i, j, 1 \leq i, j \leq m$ !, we have

$$
b_{i, j} \geq 0 \text {. }
$$

2. We cannot bribe more voters than there are. For each $i, 1 \leq i \leq m$ !,

$$
\sum_{j=1}^{m !} b_{i, j}=\left\|V_{i}\right\| .
$$

3. Altogether, we can only bribe at most $k$ people.

$$
\sum_{i=1}^{m !} \sum_{j=1}^{m !} b_{i, j}-\sum_{\ell=1}^{m !} b_{\ell, \ell} \leq k .
$$

14. A natural way of expressing this disjunction within a single integer program is to use boolean variables indicating which preference order we are concentrating on. However, this leads to an integer quadratic program. 


\begin{tabular}{|l|c|c|c|}
\hline \multirow{2}{*}{ bribery problem } & \multicolumn{3}{|c|}{ election system $\mathcal{E}$} \\
\cline { 2 - 4 } $\mathcal{E}$-bribery & plurality & approval & veto \\
$\mathcal{E}$-\$bribery & $\mathrm{P}$ & NP-complete & $\mathrm{P}$ \\
$\mathcal{E}$-weightedunary-\$bribery & $\mathrm{P}$ & NP-complete & $\mathrm{P}$ (Faliszewski, 2008) \\
$\mathcal{E}$-weighted-bribery & $\mathrm{P}$ & NP-complete & NP-complete (Brelsford et al., 2008) \\
$\mathcal{E}$-weighted- $\$$ briberyunary & $\mathrm{P}$ & NP-complete & NP-complete \\
$\mathcal{E}$-weighted- $\$$ bribery & $\mathrm{P}$ & NP-complete & NP-complete \\
& NP-complete & NP-complete & NP-complete \\
\hline
\end{tabular}

Table 1: The complexity of bribery in plurality, approval, and veto (in the setting where the number of candidates is not bounded). The results attributed to the work of Brelsford et al. (2008) and Faliszewski (2008) follow via simple arguments from results in those papers.

4. Each preference order $o_{\ell}$ disagrees with voters' preferences at least as many times as $o_{h}$. For each $\ell, 1 \leq \ell \leq m$ !, we have

$$
\sum_{i=1}^{m !} \text { agree }_{i, h}\left(\sum_{j=1}^{m !} b_{j, i}\right) \geq \sum_{i=1}^{m !} \text { agree }_{i, \ell}\left(\sum_{j=1}^{m !} b_{j, i}\right) .
$$

Clearly, each such integer program has a constant number of constraints and a constant number of variables. Thus each can be solved separately, using Lenstra's algorithm, in polynomial time. And since there are just a constant number - $m$ ! - of such integer linear programs regarding a given input, by $m$ ! applications of Lenstra's algorithm we can solve all of them. If any one of them has a feasible solution then bribery is possible and otherwise it is not.

It is interesting to consider which features of Kemeny elections allow us to employ the above attack, given that the same approach does not seem to work for either Dodgson or Young elections. One of the reasons is that the universal quantification implicit in Dodgson and Young elections is over an exponentially large search space, but the quantification in Kemeny is, in the case of a fixed candidate set, over a fixed number of options.

\section{Conclusions}

Our paper provides a study of bribery with respect to plurality rule and provides tools and results regarding many other election systems, such as scoring protocols, approval voting, and Condorcet-winner based elections. Bribery seems as important an issue as manipulation and control; our paper addresses this gap in our knowledge about the complexity of voting systems. Tables 1 and 2 collect some of the main results of this paper regarding the complexity of bribery in scoring protocols and related election systems. (However, of course, the paper contains many other results which cannot easily be presented in a form of a table, e.g., Theorem 4.19 and most of the results of Section 5.)

One of the important contributions of this paper is pointing out, by concrete examples, that NP-completeness results may not guarantee the difficulty of the most natural problem instances. In particular, Theorem 3.2 says that plurality-weighted-\$bribery is NP-complete, 


\begin{tabular}{|l|c|c|c|}
\hline \multirow{2}{*}{ bribery problem } & \multicolumn{3}{|c|}{ Scoring protocol $\alpha=\left(\alpha_{1}, \ldots, \alpha_{m}\right)}$. \\
\cline { 2 - 4 } & & $\alpha_{1}>\alpha_{2}$ and & not true that \\
$\alpha$-bribery & $\alpha_{1}=\cdots=\alpha_{m}$ & $\alpha_{2}=\cdots=\alpha_{m}$ & $\alpha_{2}=\cdots=\alpha_{m}$ \\
$\alpha$ - -bribery & $\mathrm{P}$ & $\mathrm{P}$ & $\mathrm{P}$ \\
$\alpha$-weightedunary-\$bribery & $\mathrm{P}$ & $\mathrm{P}$ & $\mathrm{P}$ \\
$\alpha$-weighted-bribery & $\mathrm{P}$ & $\mathrm{P}$ & $\mathrm{P}$ \\
$\alpha$-weighted-\$briberyunary & $\mathrm{P}$ & $\mathrm{P}$ & NP-complete \\
$\alpha$-weighted-\$bribery & $\mathrm{P}$ & NP-complete & NP-complete \\
\hline
\end{tabular}

Table 2: The complexity of bribery within scoring protocols.

but Theorem 3.8 observes that if either the weights or the prices are small enough, the problem can be solved efficiently.

Another contribution of this paper is to relate manipulation and bribery, thus making result transfer from the former to the latter a reasonable line of attack - and one that is already exploited in spirit in the proof approach of our central dichotomy result (Theorem 4.9).

As to suggested future work, we believe that studying approximation algorithms for control (by voter/candidate addition/deletion) and bribery problems currently known to be $\mathrm{NP}$-complete would be an attractive next step and we point the reader to the recent papers regarding approximation for manipulation, bribery, and control (Brelsford, 2007; Brelsford et al., 2008; Faliszewski, 2008; Zuckerman et al., 2008). It would also be interesting to study the complexity of bribery in other settings, such as with incomplete information, multiple competing bribers, or more complicated bribe structures (see the work of Faliszewski, 2008, for preliminary results on bribery with more involved pricing schemes).

\section{Acknowledgments}

We are very grateful to Samir Khuller for helpful conversations about the Bartholdi et al. (1989b) integer programming attack on fixed-candidate Dodgson elections. We are also very grateful to anonymous referees and Preetjot Singh for helpful comments. This work was supported in part by grants NSF-CCR-0311021, NSF-CCF-0426761, NSF-IIS-0713061, and AGH-UST 11.11.120.777, by Friedrich Wilhelm Bessel Research Awards to Edith Hemaspaandra and Lane A. Hemaspaandra, and by the Alexander von Humboldt Foundation's TransCoop program. This work was done in part while Piotr Faliszewski was at the University of Rochester. An early version of this paper, titled "The Complexity of Bribery in Elections," appeared in the proceedings of AAAI-06 (Faliszewski, Hemaspaandra, \& Hemaspaandra, 2006) and was also presented at COMSOC-06 and NESCAI-07.

\section{References}

Bartholdi, III, J., Tovey, C., \& Trick, M. (1989a). The computational difficulty of manipulating an election. Social Choice and Welfare, 6(3), 227-241.

Bartholdi, III, J., Tovey, C., \& Trick, M. (1989b). Voting schemes for which it can be difficult to tell who won the election. Social Choice and Welfare, 6(2), 157-165. 
Bartholdi, III, J., Tovey, C., \& Trick, M. (1992). How hard is it to control an election? Mathematical and Computer Modeling, 16(8/9), 27-40.

Betzler, N., Fellows, M., Guo, J., Niedermeier, R., \& Rosamond, F. (2008a). Fixedparameter algorithms for Kemeny scores. In Proceedings of the 4th International Conference on Algorithmic Aspects in Information and Management, pp. 60-71. SpringerVerlag Lecture Notes in Computer Science \#5034.

Betzler, N., Guo, J., \& Niedermeier, R. (2008b). Parameterized computational complexity of Dodgson and Young elections. In Proceedings of the 11th Scandinavian Workshop on Algorithm Theory, pp. 402-413. Springer-Verlag Lecture Notes in Computer Science \#5124.

Black, D. (1958). The Theory of Committees and Elections. Cambridge University Press.

Brelsford, E. (2007). Approximation and elections. Master's thesis, Rochester Institute of Technology, Rochester, NY.

Brelsford, E., Faliszewski, P., Hemaspaandra, E., Schnoor, H., \& Schnoor, I. (2008). Approximability of manipulating elections. In Proceedings of the 23rd AAAI Conference on Artificial Intelligence, pp. 44-49. AAAI Press.

Christian, R., Fellows, M., Rosamond, F., \& Slinko, A. (2007). On complexity of lobbying in multiple referenda. Review of Economic Design, 11(3), 217-224.

Condorcet, J. (1785). Essai sur l'Application de L'Analyse à la Probabilité des Décisions Rendues à la Pluralité des Voix. Facsimile reprint of original published in Paris, 1972, by the Imprimerie Royale.

Conitzer, V., \& Sandholm, T. (2006). Nonexistence of voting rules that are usually hard to manipulate. In Proceedings of the 21st National Conference on Artificial Intelligence, pp. 627-634. AAAI Press.

Conitzer, V., Sandholm, T., \& Lang, J. (2007). When are elections with few candidates hard to manipulate? Journal of the ACM, 54(3), Article 14.

Dobzinski, S., \& Procaccia, A. (2008). Frequent manipulability of elections: The case of two voters. In Proceedings of the 4th International Workshop On Internet And Network Economics, pp. 653-664. Springer-Verlag Lecture Notes in Computer Science \#5385.

Dodgson, C. (1876). A method of taking votes on more than two issues. Pamphlet printed by the Clarendon Press, Oxford, and headed "not yet published" (see the discussions in (McLean \& Urken, 1995; Black, 1958), both of which reprint this paper).

Downey, R. (2003). Parameterized complexity for the skeptic. In Proceedings of the 18th Annual IEEE Conference on Computational Complexity, pp. 147-168. IEEE Computer Society Press.

Duggan, J., \& Schwartz, T. (2000). Strategic manipulability without resoluteness or shared beliefs: Gibbard-Satterthwaite generalized. Social Choice and Welfare, 17(1), 85-93.

Dwork, C., Kumar, R., Naor, M., \& Sivakumar, D. (2001). Rank aggregation methods for the web. In Proceedings of the 10th International World Wide Web Conference, pp. 613-622. ACM Press. 
Elkind, E., \& Lipmaa, H. (2005a). Hybrid voting protocols and hardness of manipulation. In Proceedings of the 16th Annual International Symposium on Algorithms and Computation, pp. 206-215. Springer-Verlag Lecture Notes in Computer Science \#3872.

Elkind, E., \& Lipmaa, H. (2005b). Small coalitions cannot manipulate voting. In Proceedings of the 9th International Conference on Financial Cryptography and Data Security, pp. 285-297. Springer-Verlag Lecture Notes in Computer Science \#3570.

Ephrati, E., \& Rosenschein, J. (1997). A heuristic technique for multi-agent planning. Annals of Mathematics and Artificial Intelligence, 20(1-4), 13-67.

Erdélyi, G., Hemaspaandra, L., Rothe, J., \& Spakowski, H. Generalized juntas and NP-hard sets. Theoretical Computer Science. To appear.

Erdélyi, G., Hemaspaandra, L., Rothe, J., \& Spakowski, H. (2007). On approximating optimal weighted lobbying, and frequency of correctness versus average-case polynomial time. In Proceedings of the 16th International Symposium on Fundamentals of Computation Theory, pp. 300-311. Springer-Verlag Lecture Notes in Computer Science \#4639.

Erdélyi, G., Hemaspaandra, L., Rothe, J., \& Spakowski, H. (2009). Frequency of correctness versus average polynomial time. Information Processing Letters, 109 (16), 946-949.

Erdélyi, G., Nowak, M., \& Rothe, J. (2008a). Sincere-strategy preference-based approval voting broadly resists control. In Proceedings of the 33rd International Symposium on Mathematical Foundations of Computer Science, pp. 311-322. Springer-Verlag Lecture Notes in Computer Science \#5162.

Erdélyi, G., Nowak, M., \& Rothe, J. (2008b). Sincere-strategy preference-based approval voting fully resists constructive control and broadly resists destructive control. Tech. rep. arXiv:0806.0535 [cs.GT], arXiv.org. A precursor appears as (Erdélyi et al., 2008a).

Faliszewski, P. (2008). Nonuniform bribery (short paper). In Proceedings of the 7th International Conference on Autonomous Agents and Multiagent Systems, pp. 1569-1572. International Foundation for Autonomous Agents and Multiagent Systems.

Faliszewski, P., Hemaspaandra, E., \& Hemaspaandra, L. (2006). The complexity of bribery in elections. In Proceedings of the 21st National Conference on Artificial Intelligence, pp. 641-646. AAAI Press.

Faliszewski, P., Hemaspaandra, E., Hemaspaandra, L., \& Rothe, J. (2007). Llull and Copeland voting broadly resist bribery and control. In Proceedings of the 22nd AAAI Conference on Artificial Intelligence, pp. 724-730. AAAI Press. Journal version available as (Faliszewski, Hemaspaandra, Hemaspaandra, \& Rothe, 2009a).

Faliszewski, P., Hemaspaandra, E., Hemaspaandra, L., \& Rothe, J. (2008). Copeland voting fully resists constructive control. In Proceedings of the 4th International Conference on Algorithmic Aspects in Information and Management, pp. 165-176. Springer-Verlag Lecture Notes in Computer Science \#5034.

Faliszewski, P., Hemaspaandra, E., Hemaspaandra, L., \& Rothe, J. (2009a). Llull and Copeland voting broadly resist bribery and control. Journal of Artificial Intelligence Research, 35, 275-341. 
Faliszewski, P., Hemaspaandra, E., Hemaspaandra, L., \& Rothe, J. (2009b). A richer understanding of the complexity of election systems. In Ravi, S., \& Shukla, S. (Eds.), Fundamental Problems in Computing: Essays in Honor of Professor Daniel J. Rosenkrantz, pp. 375-406. Springer.

Faliszewski, P., Hemaspaandra, E., \& Schnoor, H. (2008). Copeland voting: Ties matter. In Proceedings of the 7th International Conference on Autonomous Agents and Multiagent Systems, pp. 983-990. International Foundation for Autonomous Agents and Multiagent Systems.

Friedgut, E., Kalai, G., \& Nisan, N. (2008). Elections can be manipulated often. In Proceedings of the 49rd IEEE Symposium on Foundations of Computer Science, pp. 243-249. IEEE Computer Society.

Garey, M., \& Johnson, D. (1979). Computers and Intractability: A Guide to the Theory of NP-Completeness. W. H. Freeman and Company.

Gibbard, A. (1973). Manipulation of voting schemes. Econometrica, 41(4), 587-601.

Hemaspaandra, E., \& Hemaspaandra, L. (2007). Dichotomy for voting systems. Journal of Computer and System Sciences, 73(1), 73-83.

Hemaspaandra, E., Hemaspaandra, L., \& Rothe, J. (1997). Exact analysis of Dodgson elections: Lewis Carroll's 1876 voting system is complete for parallel access to NP. Journal of the ACM, 44(6), 806-825.

Hemaspaandra, E., Hemaspaandra, L., \& Rothe, J. (2007). Anyone but him: The complexity of precluding an alternative. Artificial Intelligence, 171(5-6), 255-285.

Hemaspaandra, E., Hemaspaandra, L., \& Rothe, J. (2009). Hybrid elections broaden complexity-theoretic resistance to control. Mathematical Logic Quarterly, 55(4), 397424.

Hemaspaandra, E., Spakowski, H., \& Vogel, J. (2005). The complexity of Kemeny elections. Theoretical Computer Science, 349(3), 382-391.

Homan, C., \& Hemaspaandra, L. (2009). Guarantees for the success frequency of an algorithm for finding Dodgson-election winners. Journal of Heuristics, 15 (4), 403-423.

Kemeny, J. (1959). Mathematics without numbers. Daedalus, 88, 577-591.

Kemeny, J., \& Snell, L. (1960). Mathematical Models in the Social Sciences. Ginn.

Ladner, R., Lynch, N., \& Selman, A. (1975). A comparison of polynomial time reducibilities. Theoretical Computer Science, 1(2), 103-124.

Lenstra, Jr., H. (1983). Integer programming with a fixed number of variables. Mathematics of Operations Research, 8(4), 538-548.

Martello, S., \& Toth, P. (1990). Knapsack Problems: Algorithms and Computer Implementations. John Wiley and Sons.

McCabe-Dansted, J., Pritchard, G., \& Slinko, A. (2008). Approximability of Dodgson's rule. Social Choice and Welfare, 31(2), 311-330.

McLean, I., \& Urken, A. (1995). Classics of Social Choice. University of Michigan Press. 
Meir, R., Procaccia, A., Rosenschein, J., \& Zohar, A. (2008). The complexity of strategic behavior in multi-winner elections. Journal of Artificial Intelligence Research, 33, 149-178.

Niedermeier, R. (2002). Invitation to fixed-parameter algorithms. Habilitation thesis, University of Tübingen.

Niedermeier, R. (2006). Invitation to Fixed-Parameter Algorithms. Oxford University Press.

Papadimitriou, C. (1994). Computational Complexity. Addison-Wesley.

Procaccia, A., \& Rosenschein, J. (2007). Junta distributions and the average-case complexity of manipulating elections. Journal of Artificial Intelligence Research, 28, 157-181.

Rothe, J. (2005). Complexity Theory and Cryptology: An Introduction to Cryptocomplexity. Springer-Verlag.

Rothe, J., Spakowski, H., \& Vogel, J. (2003). Exact complexity of the winner problem for Young elections. Theory of Computing Systems, 36(4), 375-386.

Saari, D., \& Merlin, V. (2000). A geometric examination of Kemeny's rule. Social Choice and Welfare, 17(3), 403-438.

Satterthwaite, M. (1975). Strategy-proofness and Arrow's conditions: Existence and correspondence theorems for voting procedures and social welfare functions. Journal of Economic Theory, 10(2), 187-217.

Xia, L., \& Conitzer, V. (2008). A sufficient condition for voting rules to be frequently manipulable. In Proceedings of the 9th ACM Conference on Electronic Commerce, pp. 99-108. ACM Press.

Young, H. (1977). Extending Condorcet's rule. Journal of Economic Theory, 16(2), 335353.

Zuckerman, M., Procaccia, A., \& Rosenschein, J. (2008). Algorithms for the coalitional manipulation problem. Artificial Intelligence, 173(2), 392-412. 